\title{
Disséminations expérimentales d'OGM en France et en Suisse : évolution du débat public Introduction
}

Horace Perret, Barbara Bordogna Petriccione, Alain Kaufmann, Marc Audétat et Claude Joseph

\section{OpenEdition}

Édition électronique

URL : http://journals.openedition.org/ress/437

DOI : $10.4000 /$ ress.437

ISSN : 1663-4446

Éditeur

Librairie Droz

\section{Édition imprimée}

Date de publication : 1 novembre 2004

Pagination : 121-160

ISBN : 2-600-00980-9

ISSN : 0048-8046

Référence électronique

Horace Perret, Barbara Bordogna Petriccione, Alain Kaufmann, Marc Audétat et Claude Joseph, « Disséminations expérimentales d'OGM en France et en Suisse : évolution du débat public Introduction », Revue européenne des sciences sociales [En ligne], XLII-130 | 2004, mis en ligne le 06 novembre 2009, consulté le 19 avril 2019. URL : http://journals.openedition.org/ress/437 ; DOI : $10.4000 /$ ress. 437 


\author{
Horace PERRET, Barbara BORDOGNA PETRICCIONE, \\ Alain KAUFMANN, Marc AUDÉTAT et \\ Claude JOSEPH
}

\title{
DISSÉMINATIONS EXPÉRIMENTALES D'OGM EN FRANCE ET EN SUISSE : ÉVOLUTION DU DÉBAT PUBLIC
}

\section{INTRODUCTION}

Bien que variable dans le temps et dans l'espace, le débat entourant la question des organismes génétiquement modifiés (OGM) est, depuis plusieurs années, très animé dans l'espace public européen. Si diverses études ont tenté d'analyser les différentes formes prises par le débat sur cette question en Europe et aux EtatsUnis sous forme d'études isolées et d'analyses comparées, la situation en Suisse n'a mobilisé que peu d'attention ${ }^{1}$.

Dans les deux premières parties de cet article, nous nous proposons de suivre l'évolution du débat public sur les disséminations expérimentales en France et en Suisse. Par «débat public», nous entendons des échanges de points de vue et/ou des confrontations sociales qui génèrent des discussions et des délibérations dans des processus plus ou moins formels (comités d'éthique, couverture médiatique, manifestations, actions directes, etc.). De cette manière, le débat public peut être considéré comme une procédure informelle de participation du public à l'évalua-

Pour une analyse de la controverse publique aux Etats-Unis, voir: Joly P-B., Marris C., Marcant O., «La constitution du «problème public »: la controverse sur les OGM et ses incidences sur la politique publique aux Etats-Unis », volet 3, in Lemarié S., Ditner J-M., « Analyse économique du développement des cultures à base d'organismes génétiquement modifiés aux Etats-Unis», INRA-SERD, 2001. Pour une étude comparée entre France et Etats-Unis, voir: Joly P-B. et Marris C., «Mise sur agenda et controverses: une approche comparée du cas des OGM en France et aux Etats-Unis», Colloque «Risques collectifs et situations de crise. Bilan et perspectives », 7-8-9 février 2001, Auditorium du CNRS, Paris. En ce qui concerne l'Europe, une étude comparée de six pays - la France, le Royaume Unis, le Danemark, la Hollande, l'Allemagne et le Portugal - a été réalisée. Voir: Joly P-B., Assouline G., «Assessing debate and participative technology assessment in Europe, Final report», Grenoble: INRA Economie et Sociologie rurales, Tey: QAP Decision, June 2001 - (projet ADAPTA). Pour une étude spécifique du cas français, voir: Joly PB., Assouline G., Kréziak D., Lemarié J., Marris C., «L’innovation controversée: le débat public sur les OGM en France», Grenoble, INRA, 2000. En Suisse, l'influence des mouvements de consommateurs sur la mise en place du cadre légal helvétique a été brièvement analysée dans: Perrez F. X., «Taking consumers seriously: the swiss regularoty approach to genetically modified food», N.Y.U. Evironmental Law Journal, Volume 8, 2000, pp.585-604. Le débat ayant précédé le vote sur l'initiative «pour la protection génétique» a également été analysé par Buchmann M., «The impact of resistance to biotechnology in Switzerland: a sociological view of the recent referendum», in Bauer M., «Resistance to new technology: nuclear power, information technology and biotechnology », Cambridge, Cambridge University Press, 1995. 
tion des choix technologiques par opposition à des processus plus structurés comme les procédures participatives de type «conférences de consensus » (Joly \& Assouline, 2001, p.13) 2 .

La troisième et dernière partie tentera de dégager des éléments permettant de comprendre les éventuelles différences et/ou ressemblances dans le débat public des deux pays. Il faut toutefois préciser qu'une approche comparative stricte n'a pas été possible du fait de la divergence de ce qui est comparé. En France, malgré la controverse, des centaines d'essais expérimentaux ont eu lieu en plein champ et des plantes transgéniques ont été autorisées pour la culture commerciale, alors qu'en Suisse, après deux essais effectués en 1991-1992 à la faveur d'un vide juridique, douze années se sont écoulées avant qu'un nouvel essai puisse avoir lieu: celui du blé KP4 en mars 2004.

\section{LE DÉBAT PUBLIC EN FRANCE AUTOUR DES DISSÉMINATIONS EXPÉRIMENTALES}

\subsection{Introduction}

En France, la controverse publique sur les OGM ne débute véritablement qu'en 1996. Avant cette date, le dossier des OGM semble ne pas poser de problèmes. Il est entre les mains des experts, notamment celles des experts de la Commission de Génie Biomoléculaire (CGB) qui a été créée en 1986 afin d'assurer l'évaluation des plantes transgéniques. Basée sur une philosophie de progrès, elle a pour mission d'encadrer la biotechnologie afin de mieux assurer sa promotion. Ce dont elle s'acquitte avec succès puisque, depuis sa création, la France est le pays européen qui a accueilli le plus grand nombre d'essais en plein champ (Joly et al., 2000, p. 28). La France passe donc pour un pays plutôt en faveur des OGM. Et si quelques groupes d'activistes, dont Greenpeace, orientent leurs actions sur les OGM, ils n'ont encore en ce domaine aucune crédibilité et ne disposent que d'un écho limité (Joly et al., 2000, p. 27).

Mais dès 1996, au moins deux événements contribuent à faire basculer les choses. En octobre, les premiers bateaux arrivent dans les ports européens avec leur cargaison de soja transgénique en provenance des Etats-Unis, alors que l'Union européenne ne dispose encore d'aucune législation sur l'étiquetage des aliments à base d'OGM. Quelques mois plus tard, au mois de février 1997, le gouvernement Juppé refuse d'autoriser la culture du maïs Bt de Novartis en dépit de l'avis favorable de la Commission européenne, et alors que le gouvernement avait annoncé peu de temps auparavant qu'il autoriserait cette commercialisation sous réserve d'étiquetage. Cette décision provoque la démission d'Axel Kahn, alors président de la CGB, qui n'admet pas que l'avis favorable de la Commission soit ainsi remis en cause par le gouvernement. Le dossier OGM, dont la gestion avait pu être restreinte jusqu'ici à un petit cercle d'acteurs, se trouve soudainement questionné par de nouveaux venus. C'est le début d'une controverse qui

Cette distinction a servi notamment comme cadre d'analyse dans le projet ADAPTA: Joly P-B., Assouline G., op.cit. 
permettra progressivement à de nombreux groupes concernés de faire valoir leurs intérêts.

Nous allons dans cette première partie nous concentrer sur la stratégie des organisations qui ont gravité autour de la campagne anti-OGM lancée par la Confédération paysanne en 1997, car ce sont elles qui ont réussi à inscrire durablement la controverse dans l'espace public (Joly et al., 2000, p. 28). Nous prendrons comme fil conducteur la stratégie de la Confédération paysanne car elle est au centre d'un réseau de contestation à géométrie variable réunissant divers acteurs autour d'actions directes plus ou moins spectaculaires (arrachage, fauchage, incendie) visant les parcelles d'expérimentation d'OGM en plein champ $^{3}$. Nous tenterons d'évaluer dans quelle mesure ces organisations ont réussi à imposer un cadrage différent de celui qui était alors en vigueur. Par cette notion, nous nous référons à «ce qui définit, souvent implicitement, les impacts, les problèmes et les scénarios qui sont à prendre en compte, par rapport à ceux qui ne sont pas à prendre en compte et qui sont dès lors hors cadre, hors champ » (Bonneuil, 2001, p. 90).

\subsection{La stratégie de la «démonstration» ${ }^{4}$}

Nous verrons plus loin que les actions directes menées par la Confédération paysanne, mais aussi par d'autres organisations comme Greenpeace et Ecoropa dans le cadre de leur campagne anti-OGM, peuvent être lues comme autant de «démonstrations » visant à rendre visibles, via une mise en scène médiatique, les débordements technologiques qui les concernent (Callon, 2003, p. 123). Si le terme évoque en premier lieu une démonstration scientifique, de nombreuses analogies permettent de l'utiliser également dans le champ politique.

Par démonstration, nous entendons «toute action directe qui vise à rendre visible ce qui était jusqu'alors invisible par la production d'événements susceptibles d'intéresser les médias » (Barry, 2003, p. 193). Cette stratégie doit permettre à des groupes sociaux d'émerger dans l'espace public et de faire valoir certains enjeux. En prenant les médias comme témoins de leurs démonstrations, les protestataires s'efforcent de renverser un rapport de force qui leur était défavorable en tentant de démontrer une «vérité » qu'ils n'auraient pas pu démontrer autrement en public (Barry, 2003, p. 178). Si les événements reposent sur des faits solides, qu'ils résistent au filtrage des médias, cette autre version de la réalité est accréditée et se renforce (Callon, 2003, p. 211). Elle peut ensuite être discutée plus démocratiquement dans le cadre, le cas échéant, de procédures originales élaborées dans les forums hybrides 5 .

Que l'on oppose aux essais confinés qui ont lieu en laboratoires.

4 Le concept de démonstration est emprunté à Andrew Barry dans son livre, «Political Machine. Governing a technological society », London \& New York, The Athlone Press, 2001.

5 Les forums hybrides sont des procédures susceptibles de favoriser l'émergence et le suivi des controverses. Pour plus de détails, voir: Callon M., Lascoumes P. et Barthe Y., «Agir dans un monde incertain: essai sur la démocratie technique », Paris, Editions du Seuil, 2001. 


\subsection{Identités émergentes des protestataires}

La Confédération paysanne (CP) a été fondée en 1986 par José Bové, syndicaliste libertaire, et René Riesel, agriculteur et militant, en réaction à la mainmise de la Fédération nationale des syndicats d'exploitants agricoles (FNSEA) sur le monde paysan. La Confédération est un petit syndicat de gauche regroupant des paysans qui défendent une agriculture «non productiviste, solidaire et durable» ainsi que l'identité paysanne. C'est en 1997 qu'elle décide de lancer une campagne anti-OGM dont le but est prioritairement l'arrêt des expérimentations en plein champ. En somme, il s'agit pour la Confédération paysanne de contraindre le gouvernement à appliquer le principe de précaution, car, selon le syndicat paysan, «aucune barrière physique, aucune distance d'éloignement ne peuvent arrêter les transports de pollens par le vent et les insectes » (Communiqué de la Confédération Paysanne, 2003). Par ailleurs, la CP demande le maintien d'un moratoire sur la commercialisation et la mise en culture d'OGM (communiqué de la Confédération Paysanne, 2003). Il faut préciser qu'à cette époque la France a autorisé 386 disséminations expérimentales, soit 30\% de l'ensemble des disséminations autorisées dans l'Union européenne (De Cheveigné, Boy, Galloux, 2001, p. 32).

Il est important de noter qu'une démonstration, telle que nous l'avons définie, est généralement une action politique qui n'est pas le résultat de conflits idéologiques. Les identités sociales et politiques des acteurs ne préexistent pas à l'action politique, mais émergent au fil de l'action (Barry, 2003, pp. 184-85). Dans le cas qui nous occupe, on peut postuler que la campagne anti-OGM crée une certaine unité entre des acteurs qui auraient eu, en d'autres circonstances, des intérêts et des points de vue différents. Ceci est visible dès le premier arrachage d'OGM en juin 1997, la destruction d'un champ de colza résistant aux herbicides à SaintGeorges-d'Espéranche en Isère. Cet arrachage a réuni des agriculteurs, des écologistes membres de la Confédération paysanne, des Verts de l'Isère, des associations de consommateurs ainsi qu'une poignée d'élus locaux (Libération, 9 juin 1997).

La même diversité d'acteurs apparaît lors de l'action en janvier 1998 contre le maïs transgénique $\mathrm{Bt}$ de Novartis. La décision du gouvernement français, quelques mois plus tôt, d'autoriser la commercialisation de ce maïs donne lieu à deux nouvelles actions du côté des opposants: la destruction d'une partie des semences de la firme suisse par une centaine de membres de la CP, dont José Bové et René Riesel. Ces derniers investissent l'un des entrepôts de la firme près de la ville de Nérac, arrosent les semences d'eau avant de les mélanger avec des semences conventionnelles de manière à rendre toute utilisation impossible. La deuxième action est le lancement par la Confédération paysanne, l'Association des familles laïques ainsi que par une coalition de plusieurs organisations de défense de l'environnement regroupant Agir pour l'environnement, Ecoropa, Greenpeace, France Nature Environnement et Nature et Progrès d'une campagne visant à faire interdire en France la culture du maïs transgénique de Novartis (Le Monde, 30 janvier 1998). On constate ainsi que la cause anti-OGM est l'occasion de réunir, le temps d'une campagne, des associations aux intérêts a priori différents. Rien ne prédisposait en effet un parti politique comme les Verts, un syndicat paysan comme la $\mathrm{CP}$, des associations de protection de l'environnement comme Greenpeace et Ecoropa, et plus tard une association altermondialiste comme Attac 
à mener des actions communes. Une nouvelle identité émerge au fil des actions, celle d'opposant aux OGM, qui vient se greffer sur les identités préexistantes des membres de cette constellation éphémère. Le cas le plus emblématique est celui de la Confédération: quasiment inconnue avant la campagne, elle devient le symbole de la résistance aux OGM et à la mondialisation libérale, allant jusqu'à éclipser sur la scène politique française le syndicat paysan majoritaire, la FNSEA.

Il ressort également que les opposants aux OGM sont davantage unis par la nécessité d'une action directe que par une doctrine idéologique quelconque. On constate ainsi que des syndicalistes paysans, des associations altermondialistes, des députés écologiques, des élus locaux, mais aussi des paysans indiens et des personnalités du spectacle se sont joints ponctuellement aux actions menées par la Confédération. Par exemple, le 2 juin 1999, une cinquantaine de paysans indiens appartenant à une association, le KKRS («Karnakata Rajya Ryota Sangha»), de passage en France «pour dénoncer l'ordre économique mondial qui menace leur production de riz», ont rallié les militants de la Confédération et des Verts pour faucher et incendier partiellement une parcelle de colza appartenant à un organisme public, le Centre technique interprofessionnel des oléagineux métropolitains (CETIOM) (Libération, 5 septembre 2000). Le 5 juin 1999, les paysans indiens donnent un nouveau coup de main à la Confédération, mais cette fois à Montpellier, contre du riz transgénique appartenant au Centre de coopération internationale en recherche agronomique pour le développement (CIRAD). Ils pénètrent par effraction dans des serres et y détruisent des plants de riz transgéniques ainsi que du matériel informatique contenant des informations liées à cette recherche (Kempf, 2003, p. 217). Autre exemple, le 16 janvier 2003, les comédiens Christophe Malavoy, Philippe Torreton, Benoît Delepine mais aussi Anémone et Lambert Wilson, accompagnés des politiciens Noël Mamère et Harlem Désir ainsi que de Mgr Gaillot, se livrent à un arrachage de colza transgénique cultivé par le même CETIOM, sous la houlette de Greenpeace, Attac, Droits Devant! et la Confédération paysanne.

La possibilité de mobiliser des acteurs hétérogènes autour d'une action directe se vérifie également lors des nombreux procès qui émaillent la campagne antiOGM de la Confédération, dont les membres les plus actifs sont régulièrement sur le banc des accusés. La Confédération rassemble ainsi autour d'une même cause, le temps d'un procès, des témoins qui, hormis leur analyse plus ou moins critique des OGM, n'ont souvent pas grand-chose en commun. Que ce soient des chercheurs et professeurs comme Pierre-Henri Gouyon du CNRS, Arpad Pusztai, Jacques Testart de l'INRA, Gilles-Eric Séralini ou encore le spécialiste de la sécurité alimentaire Richard Lacey ou des militants d'organisations non gouvernementales (ONG) comme Arnaud Apotheker de Greenpeace et Vandana Shiva, la fondatrice de l'ONG indienne Navdanya, tous se relaient à la barre pour donner leur point de vue critique sur les OGM (Heller, 2002, pp. 16-17). Il ressort de ces exemples qu'on a bien affaire à une action politique ouverte et spontanée qui dépasse le cadre du système politique et des programmes de partis.

\subsection{Production d'événements}

Dès 1996-97, la Confédération paysanne, mais aussi Greenpeace et Ecoropa se mobilisent en tant que groupes concernés par les biotechnologies végétales et 
protestent contre les effets de ces débordements technologiques. Elles ont recours à la stratégie de la «démonstration» et tentent, ce faisant, de faire émerger dans l'espace public ce qui ne dépassait pas le cercle restreint des initiés. Comme nous allons le voir, une «démonstration » a besoin de publicité si elle veut réussir, car il lui faut produire des événements susceptibles d'être repris par les médias et rendus visibles par eux. Par ailleurs, ces événements doivent être produits sur les sites où se déroule l'action publique ou privée contestée (Barry, 2003, p. 192).

La Confédération a été très active dans ce domaine grâce à son art consommé de la mise en scène, qu'elle doit beaucoup, il faut l'avouer, à José Bové. En détruisant des parcelles expérimentales un peu partout en France en présence des médias, elle a su produire des événements sur les sites où se déroule l'action contestée. Avant leur analyse, un détour par le co-fondateur de la Confédération paysanne s'impose.

On peut distinguer deux périodes dans la campagne anti-OGM de la CP, l'avant et après démontage du Mac Donald de Millau en août 1999. Si les destructions de parcelles expérimentales réalisées durant la première période sont couvertes par les médias, c'est dans une mesure bien inférieure à celle de la deuxième période qui bénéficie de «l'effet Bové». En effet, comme le note Hervé Kempf, avant le démontage du Mac Donald, Bové est médiatiquement inconnu et, en France, l'opposition aux OGM est beaucoup plus identifiée à Greenpeace qu'à la Confédération paysanne (Kempf, 2003, p. 217). Pour comprendre l'effet Bové, il faut remonter au mois d'août 1999 et à la guerre commerciale que se livrent les Etats-Unis et l'Union européenne autour du bœuf aux hormones. Suite au refus européen d'importer le bœuf américain aux hormones, les Etats-Unis sont autorisés par l'Organisation mondiale du commerce $(\mathrm{OMC})$ à mettre en place, au titre de sanction, une taxe à l'importation de $100 \%$ sur certains produits agricoles européens, dont le roquefort. Des producteurs de lait de brebis et de roquefort de la région, dont José Bové fait partie, sont lourdement pénalisés par cette mesure. Ils décident alors de protester par une action spectaculaire. Le 12 août 1999, ils démontent le Mac Donald en construction de Millau. L'affaire, qui intervient en pleine pause estivale, fait rapidement la une des médias de l'Hexagone (Pingaud, 2002, p. 150). Le lancement d'un mandat d'arrêt contre José Bové, qui reste dans un premier temps introuvable, la mise en détention des protagonistes, la fixation de cautions étonnamment élevées pour de telles actions syndicales puis surtout la décision de José Bové de ne pas payer sa caution et de rester en prison pour défendre la liberté syndicale expliquent la tournure inattendue de cette affaire. A sa suite, José Bové devient simultanément martyr de la cause syndicale et héros national du mouvement que l'on nomme encore «anti-mondialisation ».

Trois mois plus tard, lors du sommet de l'OMC à Seattle - censé lancer en grande pompe le «Round du Millenium», un nouveau cycle de négociations commerciales - le leader syndical gagne une stature internationale grâce, cette fois, au roquefort qu'il distribue à tout va sous les caméras du monde entier. Ce coup médiatique, il le doit en partie à ses amis qui ont réussi à faire transiter incognito 300 kilos de «fromage à stries bleues» à travers la douane américaine. Seattle devient ainsi une tribune de premier choix pour le pourfendeur de la «malbouffe». Car la société civile internationale a transformé la réunion des ministres du commerce de Seattle en «happening» contestataire géant. Bové, sollicité par de nombreuses chaînes de télévision, pour qui il est l'archétype du 
résistant à la mondialisation, ne loupe pas une occasion d'associer dans les interviews la célèbre chaîne de fast food, symbole de la mondialisation, aux OGM, «tous deux produits du capital international imposés à travers le monde à toutes les cultures» (Heller, 2002, p. 31). L'épisode de Seattle a des répercussions directes sur la campagne anti-OGM de la CP. Cette dernière bénéficie dès lors sur le plan national d'une couverture médiatique sans commune mesure avec l'avant Seattle, et des slogans tels que «le monde n'est pas une marchandise» ou la terre n'est pas à vendre» gagnent en popularité auprès du public français.

Mais revenons à la «démonstration» et aux événements médiatiques qu'elle est censée produire. Ces événements peuvent prendre diverses formes. On peut notamment citer:

\subsubsection{La destruction de parcelles}

Lorsque l'on passe en revue les actions directes de la Confédération, on constate que d'une action à l'autre, leur but peut varier.

Il s'agit par exemple de:

- La protestation contre une autorisation de commercialiser le maïs génétiquement modifié de Novartis en 1998 (Bt 176);

- La dénonciation du rôle ambigu des instituts de recherche publique dans le développement des OGM. C'est le cas des actions en 1999 contre le CETIOM, le CIRAD et l'Institut national de recherche agronomique (INRA). Bové et les siens dénoncent l'implication de ces instituts dans le développement des OGM alors qu'ils sont financés par de l'argent public, et accusent la recherche d'être du même côté que les grands groupes multinationaux dans le développement d'une technique qui ne fait qu'accroître l'industrialisation de l'agriculture et la privatisation du vivant (Roy, 2001, p. 35);

- La lutte contre le risque de dissémination des OGM vers les cultures traditionnelles;

- Ou encore de la dénonciation de la confusion entretenue entre les essais proprement scientifiques - destinés à étudier les relations entre les cultures transgéniques et leur environnement - et les essais commerciaux, destinés à vérifier l'adaptation des variétés transgéniques aux conditions locales.

Malgré cette diversité, ces actions s'inscrivent toutes dans une volonté plus générale de démontrer qu'une autre agriculture est possible, différente de l'agriculture industrielle dont les OGM sont le dernier avatar: une agriculture paysanne, durable et solidaire. Il s'agit pour le syndicat paysan de contester une technologie jugée dangereuse pour les paysans et la société, et de rendre visible ce qui était jusqu'alors invisible, i.e. le fait que les OGM sont dans les champs et qu'ils pourraient contaminer les cultures traditionnelles si leurs pollens venaient à se disséminer.

Mais les destructions de parcelles n'auraient pas le même impact si elles n'étaient savamment mises en scène. On peut relever les aspects suivants:

- l'aspect souvent festif que donnent les organisateurs aux destructions de parcelles. Il n'est pas rare qu'un casse-croûte soit organisé sur les lieux de la destruction; 
- les opposants agissent la plupart du temps à visages découverts au risque de poursuites judiciaires. C'est le cas lors de la campagne de l'été 2001 qui vise à dénoncer les risques de dissémination de pollens, qui avaient été confirmés par un rapport de l'Agence française de sécurité sanitaire des aliments ${ }^{6}$ (Le Monde, 23 août 2001).

- le dépôt symbolique de la «récolte» (les plantes fauchées) devant les préfectures concernées (préfectures de la Drôme, de Nîmes, mairie de Bagnolet en 2003).

La presse audiovisuelle et la presse écrite sont bien évidemment invitées sur les lieux de l'action. Lors de la destruction du maïs de Novartis en 1998, des équipes de France 2 et France 3 sont présentes (Roy, 2001, p. 24). Autre exemple, déjà cité, l'arrachage qu'effectue au mois de janvier 2003 un groupe de personnalités françaises connues du grand public regroupant des comédiens, politiciens et religieux, auquel un envoyé spécial du journal Libération est invité (Libération, 17 janvier 2003).

\subsubsection{Les procès}

Les destructions des parcelles d'OGM étant une transgression de l'ordre légal, elles sont suivies par des procès. Ces procès sont tout aussi bien exploités que les destructions par la CP qui s'efforce de les instrumentaliser à son avantage. On peut faire l'hypothèse que les procès sont transformés en événements médiatiques par le syndicat paysan qui utilise plusieurs procédés à cette fin. De nombreux incidents plus ou moins organisés viennent ainsi enrayer le bon déroulement de la justice. Lors du procès à Montpellier en 2001 pour l'action contre le CIRAD, trois cents manifestants pénètrent dans le tribunal et interrompent l'audience en scandant des slogans de soutien à José Bové. Un «forum citoyen» est par ailleurs organisé par un collectif anti-OGM parallèlement au procès pour l'action contre le CETIOM à Foix afin de sensibiliser les citoyens aux OGM, qui réunit deux mille personnes (Le Monde, 7 septembre 2000) 7 .

Mais les procès jouent surtout la fonction de caisse de résonance pour le discours de la CP. Dès le procès de février 1998 pour la destruction des semences de Novartis, les prévenus - José Bové, René Riesel et Francis Roux - annoncent leur intention d'aller au-delà des faits qu'on leur reproche. Ils entendent en effet «faire de leur procès celui des plantes transgéniques en général, du système d'évaluation des risques et du processus de prise de décision qui avait conduit à

Ce rapport a relevé des très faibles traces d'OGM (de l'ordre de 0,1\%) dans $41 \%$ d'échantillons de maïs conventionnel testés, malgré l'instauration d'un moratoire sur les nouvelles variétés de plantes en 1998. Après avoir demandé sans succès au gouvernement de faire en sorte que les essais aient lieu en site confiné et non en plein champ, le syndicat paysan met sa menace à exécution et prend lui-même en charge la destruction d'essais en plein champ au nom du principe de précaution.

${ }^{7}$ Le paroxysme de cette stratégie est atteint lors du procès pour le démontage du McDo fin juin 2000 à Millau. La CP a décidé d'en faire le procès de la mondialisation. Une gigantesque fête rassemblant 50'000 personnes sous la bannière de l'anti-mondialisation est organisée dans les rues de Millau où concerts et forums se mêlent durant un week-end festif (Le Monde, 3 juillet 2000). 
l'autorisation prématurée du maïs transgénique en question avant que ne soit organisé le débat promis par le gouvernement» (Roy, 2001, p. 24). L'intention est donc affichée d'utiliser les procès comme des tribunes politiques afin de susciter un débat public autour des OGM. Les actions de l'été 1999 contre les instituts de recherche publique - le CETIOM et le CIRAD - avaient pour but de dénoncer les liens croissants entre la recherche publique et les multinationales du secteur agrochimique. Les procès qui suivent ces actions sont l'occasion pour la CP d'entamer un débat autour des relations entre recherche publique et marché. Si dans un premier temps l'incompréhension des chercheurs est totale face à ces actions - ils ne comprennent pas qu'on puisse s'en prendre à des recherches dont le but est justement la constitution d'une contre-expertise sur les risques potentiels des OGM -, la situation semble évoluer en cours de route. En juillet 2003, dans une lettre de soutien à José Bové, alors incarcéré, adressée au Président de la République, un nombre important de chercheurs appartenant à ces organismes ${ }^{8}$ déclare que «la société civile doit être partie prenante des décisions concernant ses objectifs et l'utilisation de ses résultats». Ils reconnaissent ainsi que la recherche peut faire l'objet d'un débat public. Ils déclarent également dans cette lettre que les actions de Bové et les siens ont «rempli une fonction d'alerte», et que «la gravité du problème de l'utilisation des OGM en a largement bénéficié » (Le Monde, 28 juin 2003).

Mais le débat ne s'arrête pas aux OGM ni aux interactions entre la science et le marché, il traite des limites de l'agriculture productiviste et des conséquences des OGM sur l'agriculture des pays du Sud. Ce lien est notamment fait par la militante indienne Vandana Shiva lors de son intervention en février 1998 à Agen en sa qualité de témoin au procès pour la destruction des semences Novartis. Ce lien avec le Sud, la CP le crée aussi délibérément par l'action contre le CIRAD en 1999, déjà citée, à laquelle participent une cinquantaine de paysans indiens. Pour Bové, «la problématique du riz transgénique doit permettre en effet de démontrer la menace commune que font peser les OGM sur les paysans du Sud ou du Nord» (Kempf citant Bové, 2003, p. 216). Le contexte est toutefois différent, puisqu'il s'agit là d'une action directe et non d'un procès.

L'acte de convoquer à la barre des témoins plus ou moins connus du grand public et détenteurs d'une contre-expertise participe également à la production de l'événement et à sa mise en scène. Mais il peut aussi être interprété comme une volonté indirecte de donner de la «robustesse» à l'événement en question. Pour qu'un événement soit robuste, il doit pouvoir résister aux questions qu'on lui pose, aux critiques qu'on lui adresse, aux enquêtes auxquels il serait soumis, le succès d'une « démonstration » dépendant de la capacité d'un événement à résister à cet examen. Si l'événement qui est démontré n'est pas assez robuste, la «démonstration» et avec elle la revendication politique s'évanouissent (Callon, 2003, p. 213). En l'occurrence, le syndicat paysan utilise cet espace public pour y

Il s'agit de l'INRA, du CIRAD, de l'INSERM et de l'IRD. Rappelons le contexte de cette lettre de soutien: José Bové est incarcéré depuis le 22 juin 2003 pour une peine de dix mois qui est le résultat de deux condamnations: l'une de huit mois avec sursis, prononcée par le tribunal d'Agen en 1998 pour la destruction de semences transgéniques, réduite de moitié en février 2003. Et l'autre de six mois, infligée en décembre 2001 par la cour d'appel de Montpellier pour des faits similaires (Libération, 23 juin 2003). 
délivrer son message réitérant la possibilité d'un monde sans OGM. Le tribunal joue alors la fonction d'un «mini espace public » où l'événement - la destruction de parcelles expérimentales - peut gagner en objectivation par le jeu des arguments et contre arguments, relayés par les médias.

\subsubsection{D'autres actions directes}

Greenpeace recourt elle aussi à la stratégie de la «démonstration». Elle est aguerrie aux actions coup de poing qui sont autant d'événements à l'intention des médias. On peut même dire qu'elle est la première à lancer l'alerte, puisque sa campagne anti-OGM débute en 1996 déjà. Le 6 novembre 1996, elle intercepte la première cargaison de soja transgénique qui arrive à Hambourg. Une de ses équipes parvient à retarder le bateau dans le port le temps de lui imprimer le slogan «we are not your guinea pigs» (nous ne sommes pas vos cobayes) sur la coque (Kempf, 2003, p. 136). L'association de protection de l'environnement s'en prend également au colza transgénique qui, en 1996, vient d'être autorisé en Europe. Elle invoque un risque environnemental, plus précisément le fait qu'on ne connaît pas avec précision les probabilités de dissémination des gènes de résistance aux herbicides (Joly et al., p. 53, 2000). Par la suite, l'association crée à nouveau des événements médiatiques, lorsqu'elle se mobilise contre le maïs $\mathrm{Bt}$ 176 de Novartis. Elle organise par exemple des manifestations devant les locaux de Novartis près de Toulouse aux mois de février et de décembre 1997, lorsque le gouvernement s'apprête à donner son autorisation au maïs de la firme suisse. En septembre 1998, les militants écologistes récoltent plusieurs tonnes de maïs transgénique et le déversent devant les locaux de la firme à Bâle. Les risques soulevés par Greenpeace concernant ce maïs sont le risque environnemental que pose le problème de l'acquisition de résistance à la pyrale et le risque pour la santé humaine (Joly et al., pp. 53-54, 2000).

\subsection{Bilan}

La pression exercée par la Confédération sur le gouvernement depuis 1997 a permis d'obtenir quelques résultats concrets. Dès 1999, le contexte change, puisque l'Union européenne décrète un moratoire de facto sur toute nouvelle autorisation de mise en culture et de mise sur le marché d'OGM. Bové n'hésite pas à déclarer à un journaliste que «c'est après nos premiers arrachages en 1999 que l'Europe a décrété son moratoire sur les cultures OGM» (Libération, 23 octobre 2002). Il ne nous semble toutefois pas possible d'établir un lien causal direct entre cette mesure et les destructions de parcelles, bien que la France ait joué un rôle clé dans l'adoption de ce moratoire. En revanche, la campagne de l'été 2001 a clairement permis de démontrer la confusion entretenue entre les essais proprement scientifiques, destinés à étudier les relations entre les cultures transgéniques et leur environnement, et les essais commerciaux, destinés à vérifier l'adaptation des variétés transgéniques au terroir. Cette série d'arrachages a conduit à l'organisation d'un débat public ${ }^{9}$ en 2002 qui a conclu à la nécessité

Ce débat s'est tenu les 4 et 5 février 2002. Il a réuni pendant deux jours experts et partisans des deux bords sous la direction de quatre «sages» désignés par le gouvernement: Jean-Yves Le Déaut, président de l'Office parlementaire des choix technologiques, Jacques Testart, président du 
d'encadrer très strictement les essais et à considérer leur utilité socio-économique (Kempf, 2003, p. 231). Par ailleurs, la campagne du syndicat paysan a eu un effet sur la stratégie de certaines sociétés agrochimiques. L'allemande Bayer CropScience ou la française Biogemma ${ }^{10}$, toutes deux victimes d'arrachages, hésitent désormais à poursuivre leurs essais en plein champ sur le territoire français. La Confédération a également contribué à faire entrer la question des OGM dans le débat public. En France, il ne se passe quasiment pas une semaine sans qu'un journal n'aborde les enjeux et les questions posés par les OGM ${ }^{11}$. Mais selon nous son influence majeure est d'avoir réussi à modifier les termes mêmes du débat, comme nous le verrons plus loin.

\section{LE DÉBAT PUBLIC EN SUISSE AUTOUR DU BLÉ KP4}

En Suisse, le débat relatif aux OGM a débuté en 1993-1994 avec le lancement de l'initiative «Pour la protection génétique» qui a été soumise au vote populaire le 7 juin 1998. Depuis lors, la seule dissémination de plante transgénique qui ait eu lieu sur le territoire suisse est précisément celle très récente du blé KP4 de l'EPFZ. Avant cela, les seules disséminations de plantes transgéniques jamais effectuées en Suisse avaient été réalisées en 1991-1992 à la faveur d'un vide juridique par la Station fédérale de recherche en production végétale de Changins.

En effet, depuis 1998, trois demandes de dissémination expérimentale ont été déposées en Suisse. La première concerne un maïs tolérant à l'herbicide glufosinate de l'entreprise Plüss-Staufer $A G^{12}$. La seconde est celle de la Station fédérale de recherche en production végétale de Changins relative à une pomme de terre résistante à une maladie fongique, le mildiou ${ }^{13}$. Enfin la troisième demande concerne une variété de blé transformée pour résister à une autre maladie

Commission française du développement durable, Didier Sicard, président du Comité national d'éthique, et Christian Babusiaux, président du Conseil national de l'alimentation. Son but était de déterminer s'il fallait poursuivre les essais en plein champ malgré la campagne d'arrachages de la CP (Le Monde, 7 février 2002).

1o Le 19 juillet 2003, une parcelle expérimentale de maïs transgénique appartenant à Bayer CropScience était fauchée par des membres de la CP, d'Attac, des Verts, demandant, entre autres, la libération de José Bové. Les mêmes avaient procédé à la destruction de plants de maïs transgénique appartenant à Biogemma au mois de juillet 2001. Selon la société française, la parcelle qui avait fait l'objet d'un arrachage n'était pas un maïs génétiquement modifié pour être résistant à un herbicide, mais un nouveau système d'hybridation devant réduire les coûts de production (Figaro du 24 juillet 2003 et Libération du 28 août 2001).

11 Le nombre d'article du journal Le Monde contenant le mot clé «OGM» est à cet égard révélateur. Il ne cesse d'augmenter de 1996, date à partir de laquelle les premières cargaisons de soja transgénique arrivent en Europe, à 2000, année où la campagne de la CP bat son plein. Dès cette date, il se stabilise, toutefois à un niveau légèrement inférieur. Ainsi, de 6 articles en 1996, on est passé à 13 en 1997 puis à 79 en 1998, avant de connaître une explosion en 1999 avec 202 articles. Le record est atteint en 2000 avec 230 articles, avant de diminuer successivement en 2001 et 2002 avec respectivement 209 et 170 articles. En 2003, le nombre d'articles contenant le mot clé «OGM» repart à la hausse, puisqu'il atteint 222 (ces résultats ont été obtenus à l'aide d'une recherche sur le site Europresse: http://www.bpe.europresse.com/Mainsite/Frames/FrameMain.asp).

12 Demande déposée le 11 novembre 1998.

13 Demande déposée le 9 décembre 1999. 
fongique, la carie du blé, qui a été déposée par l'Institut de biologie végétale de l'Ecole polytechnique fédérale de Zurich (EPFZ) ${ }^{14}$. Les deux premières ont été refusées en avril 1999 et la troisième a finalement été acceptée et le blé transgénique a pu être planté le 18 mars 2004, presque trois ans et demi après le dépôt de la demande le 15 novembre 2000.

La recherche en biotechnologies a été promue au début des années 90 par le lancement d'un Programme prioritaire en biotechnologie (PPB) voté par le parlement. Ce programme visait à promouvoir la compétitivité de la place helvétique dans un modèle classique de type «technology push». La durée du PPB était de 10 ans, et c'est dans ce cadre que les demandes de dissémination concernant le blé transgénique de l'EPFZ ainsi que celle concernant la pomme de terre résistante au mildiou ont été effectuées.

\subsection{Le cadre légal}

Nous allons maintenant donner un aperçu du cadre légal sous-tendant la controverse sur la dissémination expérimentale du blé KP4 et identifier les parties prenantes à cette controverse ainsi que leurs arguments.

Dès le début des années 1990, face aux avancées rapides dans le domaine des biotechnologies, la législation suisse se révèle insuffisante pour encadrer correctement le développement et l'utilisation de cette nouvelle technologie. Une initiative dite «Pour la protection génétique» est lancée en 1993 proposant un cadre très restrictif dans les domaines à la fois médical et agricole. Par ailleurs, une motion demandant l'établissement rapide d'une législation en matière de génie génétique dans le domaine non humain (motion Gen-Lex) fut déposée au Conseil national en 1996. Elle permettait de garantir aux citoyens que les lacunes de la législation seraient comblées sans qu'il soit pour cela nécessaire d'accepter l'initiative «Pour la protection génétique», jugée majoritairement trop restrictive par le gouvernement, en particulier au niveau des limites posée à la recherche médicale.

Pendant ce temps, des dispositions relatives au génie génétique dans le domaine non humain étaient introduites dès 1995 dans la loi sur la protection de l'environnement (LPE) ${ }^{15}$, dispositions qui furent précisées en 1999 par deux ordonnances: l'Ordonnance sur la dissémination dans l'environnement (ODE) et l'ordonnance sur l'utilisation confinée (OUC). Le but de la LPE est de protéger les hommes et l'environnement des atteintes nuisibles ${ }^{16}$. Les dispositions spécifiques de la LPE relatives aux organismes dangereux pour l'environnement ainsi que l'ODE ont naturellement les mêmes objectifs ${ }^{17}$. La

14 Demande déposée le 15 novembre 2000.

15 Art. 29 concernant les organismes dangereux pour l'environnement; cette article a été introduit le 21 décembre 1995 et est entré en vigueur le $1^{\text {er }}$ juillet 1997. Il contient notamment des dispositions relatives aux disséminations à titre expérimental (art. 29e) et à la mise en place d'une commission d'experts pour la sécurité biologique (art. 29h).

${ }_{16}$ La présente loi a pour but de protéger les hommes, les animaux, les plantes, leurs biocénoses et leurs biotopes des atteintes nuisibles ou incommodantes, et de conserver la fertilité du sol (art.1 LPE).

17 Voir art. 29 LPE et art. 1 ODE. 
réglementation concernant les disséminations expérimentales vise donc uniquement à éviter les risques pour l'homme et pour l'environnement. La loi ne mentionne pas d'autre paramètre en fonction duquel évaluer cette activité. Ces dispositions ne remplissaient toutefois pas encore le mandat législatif conféré par l'art. 24 novies, al. 3 de la Constitution, que le Parlement s'était par ailleurs engagé à compléter le plus rapidement possible en adoptant la motion Gen-Lex. Des lacunes subsistaient en particulier en ce qui concerne «le respect de la dignité de la créature», «la protection et la conservation de la diversité biologique et l'utilisation durable de ses éléments constitutifs » ainsi que la question de la responsabilité.

Deux commissions d'experts furent en outre créées. La première, la Commission fédérale d'experts pour la sécurité biologique (CFSB), fut instituée en 1996 et chargée en particulier d'évaluer les demandes d'autorisation pour l'utilisation en milieu confiné, les disséminations expérimentales et la mise dans le commerce d'organismes génétiquement modifiés ou pathogènes du point de vue de la sécurité biologique ${ }^{18}$. La seconde, la Commission fédérale d'éthique dans le domaine non humain (CENH), fut mise en place en avril 1998 avec pour mandat de donner son avis d'un point de vue éthique sur l'observation de divers principes ${ }^{19}$. Elle fut en particulier chargée de conseiller le Conseil fédéral et les autorités et de donner son avis d'un point de vue éthique concernant la production et l'utilisation en milieu confiné, les disséminations expérimentales et la mise dans le commerce d'organismes génétiquement modifiés ou pathogènes.

Le 7 juin 1998, l'initiative dite «Pour la protection génétique» ayant été refusée par le peuple, les travaux des chambres fédérales sur le projet de cadre légal en matière de génie génétique dans le domaine non humain (projet Gen-Lex) se sont poursuivis. Ce n'est qu'en 2003 toutefois que le projet Gen-Lex a abouti sous forme d'une loi spécifique réglementant l'utilisation du génie génétique dans le domaine non humain (Loi génie génétique - LGG du 21 mars 2003). La LGG a intégré et complété les dispositions de la loi sur la protection de l'environnement (LPE) de 1995 de même que celles de l'ordonnance sur l'utilisation confinée (OUC) et de l'ordonnance sur la dissémination dans l'environnement (ODE). Un droit de recours a été conféré aux associations en ce qui concerne la mise en circulation d'organismes génétiquement modifiés destinés à être utilisés dans l'environnement. En outre, les dispositions de l'Union européenne ainsi que les exigences du Protocole de Carthagène sur la prévention des risques biotechnologiques ont été prises en compte et la question de la responsabilité juridique clarifiée. La LGG est entrée en vigueur en janvier 2004. Les dispositions concernant la mise en place d'un moratoire de cinq ans sur la dissémination à but commercial de plantes génétiquement modifiées n'ayant finalement pas été intégrée dans la

Ordonnance sur la Commission fédérale d'experts pour la sécurité biologique du 20 novembre 1996.

19 Principes du respect de la dignité de la créature, de la protection de la sécurité de l'homme et de l'environnement, de la protection de la diversité génétique des espèces animales et végétales et de leur exploitation durable. (Décret du Conseil fédéral du 27 avril 1998 instituant la commission fédérale d'éthique pour le génie génétique dans le domaine non humain) 
LGG, une initiative affichant la même revendication a été lancée en février 2003 par diverses associations et partis ${ }^{20}$.

La procédure d'autorisation pour les disséminations expérimentales, sous le coup de laquelle tombe la demande déposée par l'EPFZ le 15 novembre 2000 pour le blé KP4, est réglementée par l'ordonnance sur les disséminations expérimentales (ODE). L'Office fédéral de l'environnement, des forêts et du paysage (OFEFP) est l'organe compétent pour délivrer une telle autorisation (art. 7). Plus loin, l'article 19 précise les conditions d'octroi d'une autorisation et stipule que l'OFEFP doit évaluer et examiner la demande en tenant compte des avis reçus, notamment de la part de la Commission fédérale pour la sécurité biologique (CFSB) et du service spécialisé du canton (art. 19.1 ODE) qui, dans le cas du blé KP4, est le Service spécialisé pour la sécurité biologique du Service des déchets, de l'eau, de l'énergie et de l'air (AWEL) ${ }^{21}$. L'autorisation est octroyée à condition que, d'une part «l'évaluation de la demande, en particulier l'évaluation du risque, établit que, d'après l'état de la science et l'expérience, la dissémination expérimentale ne peut pas mettre en danger l'homme et l'environnement» (art. 19.1.a ODE) et d'autre part que «L'Office vétérinaire fédéral (OVF), l'Office fédéral de la santé publique (OFSP), l'Office fédéral de l'agriculture (OFAG) approuvent la réalisation de la dissémination expérimentale sur la base de l'évaluation de la demande, en particulier de l'évaluation du risque » (art. 19.1.b ODE).

\subsection{Les parties prenantes}

Les parties directement impliquées dans le parcours sinueux de cette demande d'autorisation sont de plusieurs types. En premier lieu, certaines autorités officielles sont clairement définies comme étant appelées à s'exprimer sur une telle demande. En effet, une fois déposée auprès de l'OFEFP, l'organe en charge de délivrer les autorisations de dissémination d'OGM, la demande fait l'objet d'un processus de consultation au cours duquel elle est examinée par divers départements et commissions. L'OVF, l'OFSP, l'OFAG, la Commission pour la sécurité biologique (CFSB), la Commission fédérale d'éthique pour le génie génétique dans le domaine non humain $(\mathrm{CENH})$ et l'AWEL du canton de Zurich ont ainsi donné leur avis concernant cette demande. L'OFEFP rend ensuite sa décision contre laquelle il peut être fait recours auprès du Département fédéral de l'environnement, des transports, de l'énergie et de la communication (DETEC), à la tête duquel se trouve le conseiller fédéral socialiste Moritz Leuenberger. L'OFEFP est en effet administrativement dépendante du DETEC dont elle constitue l'un des offices. Enfin, en dernière instance, il peut être fait recours auprès du Tribunal fédéral contre la décision prise par le DETEC.

En ce qui concerne la société civile, les associations et mouvements étant intervenus au cours de la procédure d'autorisation provenaient d'horizons variés:

L'initiative «Pour des aliments produits sans manipulations génétiques», dite initiative Stop OGM a été lancée le 18 février 2003 et en septembre 2003 elle avait déjà récolté les 100'000 signatures nécessaires pour pouvoir être soumise au vote populaire.

21 Le nom allemand (complet et exact) est Bauderdirektion des Kanton Zürich, Amt für Aball, Wasser, Energie und Luft (AWEL), KSF/Fachstelle für biologische Sicherheit. 
- En premier lieu, les riverains du site d'expérimentation à Lindau dans le canton de Zurich ont formé le groupe de travail «Lindau gegen GenetechWeize» (Lindau contre le blé transgénique) afin de s'opposer à cet essai.

- L'organisation de défense de l'environnement Greenpeace s'est également fortement mobilisée sur ce dossier, mais, à la différence des riverains, son action n'est nullement ponctuelle et fait au contraire partie d'une campagne internationale contre les OGM lancée par Greenpeace en 1996 déjà. D'autres organisations écologistes telles que Pro Natura, les Verts suisses et l'appel de Bâle contre le génie génétique se sont également manifestées à diverses reprises.

- Diverses organisations paysannes nationales et locales se sont mobilisées au rang desquelles les agriculteurs bio zurichois et schaffousois, l'association de paysans de production intégrée IP-Suisse et l'association des petits et moyens agriculteurs VKMB.

- L'association de consommateurs KAGfreiland ${ }^{22}$ ainsi qu'une association active dans la coopération Nord-Sud, Swissaid, se sont également mobilisés contre cette dissémination.

Enfin, au niveau de la recherche scientifique, le premier acteur impliqué est le Professeur Sautter et son équipe à l'Institut de biologie végétale de l'EPFZ, qui sont à l'origine de la demande de dissémination du blé KP4. Ces recherches étant publiques, il convient de mentionner également l'organe ayant décidé de soutenir financièrement ce projet, à savoir le Fonds national pour la recherche scientifique (FNS), département IV. Comme déjà mentionné, le blé transgénique fait en effet partie du Programme prioritaire biotechnologie (PPB) qui s'est étalé sur la période 1991-2001.

\subsection{Les arguments du débat}

Pour analyser les arguments mobilisés par les parties prenantes dans le débat public, nous recourrons au concept d' «arène». Emprunté à la science politique, il désigne les espaces symboliques de confrontation où se discutent les problèmes publics, selon des règles du jeu prédéfinies (Joly \& Marris, 2002, p. 46). Une arène - qu'elle soit politique, économique, médiatique, légale, religieuse ou scientifique - se caractérise notamment par un cadre référentiel spécifique (ou référent symbolique), la prédominance d'un certain type d'acteurs et par un type de ressources qui lui sont propres. Ainsi, l'arène politique a pour cadre référentiel les valeurs démocratiques, pour acteurs dominants la société civile (citoyens individuels ou associations) et les politiciens et pour ressources le pouvoir ainsi que la confiance.

${ }^{22}$ Konsument/innen-Arbeitsgruppe für tier- und umweltfreudliche Nutztierhaltung 
Le concept d'arène: quelques caractéristiques générales ${ }^{23}$.

\begin{tabular}{|l|l|l|l|l|l|}
\hline Arène & Dispositif & Ressources & $\begin{array}{l}\text { Référent } \\
\text { symbolique }\end{array}$ & $\begin{array}{l}\text { Acteurs } \\
\text { dominants }\end{array}$ & Production \\
\hline Economique & Marché & $\begin{array}{l}\text { Argent, } \\
\text { image }\end{array}$ & $\begin{array}{l}\text { Efficience, } \\
\text { transactions }\end{array}$ & $\begin{array}{l}\text { Producteurs, } \\
\text { Consom- } \\
\text { mateurs }\end{array}$ & Produits \\
\hline Scientifique & $\begin{array}{l}\text { Laboratoires, } \\
\text { institutions } \\
\text { scientifiques }\end{array}$ & $\begin{array}{l}\text { Preuve } \\
\text { scientifique, } \\
\text { méthode, } \\
\text { réputation }\end{array}$ & $\begin{array}{l}\text { Vérité, } \\
\text { rationalité, } \\
\text { rigueur, } \\
\text { impartialité }\end{array}$ & $\begin{array}{l}\text { Scientifiques, } \\
\text { experts, } \\
\text { profanes }\end{array}$ & $\begin{array}{l}\text { Connaissances, } \\
\text { expertise }\end{array}$ \\
\hline Réglementaire & $\begin{array}{l}\text { Autorités } \\
\text { administra- } \\
\text { tives }\end{array}$ & $\begin{array}{l}\text { Règles, } \\
\text { codes, } \\
\text { procédures }\end{array}$ & $\begin{array}{l}\text { Contrôle, } \\
\text { indépen- } \\
\text { dance }\end{array}$ & $\begin{array}{l}\text { Experts, } \\
\text { juristes }\end{array}$ & $\begin{array}{l}\text { Régulation, } \\
\text { normes }\end{array}$ \\
\hline $\begin{array}{l}\text { Juridique } \\
\text { ou légale }\end{array}$ & $\begin{array}{l}\text { Cours } \\
\text { de justice }\end{array}$ & $\begin{array}{l}\text { Lois, } \\
\text { procédure }\end{array}$ & Justice & $\begin{array}{l}\text { Juges, } \\
\text { avocats }\end{array}$ & Jurisprudence \\
\hline Politique & $\begin{array}{l}\text { Parlements, } \\
\text { rue }\end{array}$ & $\begin{array}{l}\text { Pouvoir, } \\
\text { confiance }\end{array}$ & Démocratie & $\begin{array}{l}\text { Politiciens, } \\
\text { Citoyens, } \\
\text { associations }\end{array}$ & Lois, valeurs \\
\hline Médiatique & $\begin{array}{l}\text { Journaux, } \\
\text { télévision, } \\
\text { radio }\end{array}$ & $\begin{array}{l}\text { Audience, } \\
\text { sources }\end{array}$ & $\begin{array}{l}\text { Information, } \\
\text { vérité, } \\
\text { liberté } \\
\text { d'expression }\end{array}$ & $\begin{array}{l}\text { Journalistes, } \\
\text { audience }\end{array}$ & $\begin{array}{l}\text { Articles } \\
\text { Productions } \\
\text { audio-visuelles }\end{array}$ \\
\hline
\end{tabular}

Selon cette approche, l'intensité des débats est proportionnelle au nombre d'arènes mobilisées par le problème en question. Ce n'est que lorsqu'un débat mobilise plus de deux arènes et qu'il y a un nombre croissant d'interactions entre arènes que l'on peut parler de controverse publique. Dans ce cas, on assiste à l'intrusion d'acteurs dans des arènes où ils ne sont normalement pas influents (Joly \& Assouline, 2001, pp. 22-26). Dans le cas qui nous intéresse, il est possible de classer les parties prenantes à la controverse concernant la demande de dissémination du blé KP4 de l'EPFZ en quatre grandes arènes: l'arène scientifique, l'arène de régulation, l'arène juridique et l'arène politique.

La demande de dissémination expérimentale de blé transgénique résistant à la carie de l'EPFZ fut déposée le 15 novembre 2000 auprès de l'OFEFP. La demande portait sur une surface de $90 \mathrm{~m}^{2}$ dont $8 \mathrm{~m}^{2}$ effectivement plantés avec le blé transgénique. La période d'essai envisagée allait du mois de février 2002 au mois d'avril 2003. Trois transgènes avaient été insérés dans ce blé: un gène de résistance à l'herbicide phosphinothricine (gène bar), un gène de résistance à l'antibiotique ampicilline (gène bla) et un gène d'un virus ARN attaquant le champignon responsable de la carie du blé (gène $k p 4$ ). Considéré comme incomplet, le dossier fut retourné à son expéditeur qui le déposa une nouvelle fois complété le 4 janvier 2001.

23 Librement adapté de Joly P.-B. et Assouline G. (projet ADAPTA), op. cit., p. 24. 
Lorsqu'elle fut publiée dans la Feuille fédérale le 13 février 2001, la demande de dissémination expérimentale de l'EPFZ n'a suscité que quelques réactions critiques $^{24}$. Le débat autour de cette dissémination, bien que suivi de très près notamment par ses opposants, est en effet resté peu intense durant toute la procédure de consultation jusqu'au refus de l'OFEFP le 20 novembre 2001. Une certaine mobilisation était certes perceptible à l'occasion de la soirée d'information au public tenue le 21 février 2001 dans la commune de Lindau ${ }^{25}$, mais la plus grande activité se déroulait à cette période au sein des diverses instances appelées à donner leur avis sur cette dissémination. Entre mars et octobre 2001, toutes transmirent leur position à l'OFEFP.

Ayant examiné la demande du point de vue des risques possibles pour l'homme et l'environnement, la CFSB jugea que:

«l'essai en plein champ projeté peut être considéré comme sûr en raison du site sur lequel il se déroule, de la dimension de la superficie cultivée $\left(8 \mathrm{~m}^{2}\right)$ et des caractéristiques de l'essai (recherche fondamentale pure) et qu'il ne comporte pas de risque fondamental pour l'homme et l'environnement. ${ }^{26}$

On peut noter que l'ensemble de l'argumentation avancée par la CFSB relève d'un cadre de référence scientifique (risques pour la santé et l'environnement) avec une évaluation à la fois de la plante de blé en elle-même, du site de dissémination et de l'aspect sécurité. Sur l'aspect sécurité, il faut relever qu'un point est consacré à l'information du public: la possibilité donnée à la population de poser des questions durant la soirée d'information qui a eu lieu durant la phase préparatoire doit être poursuivie durant la période de réalisation de l'essai ${ }^{27}$.

Au niveau du canton de Zurich, l'AWEL, ainsi que les autres services consultés ${ }^{28}$ ont considéré que l'essai pouvait être autorisé sous certaines conditions. Les trois autres Offices fédéraux appelés à se prononcer sur cette demande - l'Office fédéral pour la santé publique (OFSP), l'Office fédéral pour l'agriculture (OFAG) et l'Office vétérinaire fédéral $(\mathrm{OVF})$ - ont tous conclu à une absence de risques dans leur domaine de compétence et n'ont donc pas soulevé d'objection à la tenue de l'essai. On peut noter que, en examinant la demande du point de vue essentiellement de la biosécurité, à savoir les risques éventuels pour la santé et l'environnement, toutes ces instances consultatives sont restées fidèles au cadre de référence scientifique.

Quant à la $\mathrm{CENH}$, une majorité de ses membres arriva à la conclusion que, d'un point de vue éthique, l'essai pouvait être réalisée ${ }^{2}$. En portant son analyse sur

24 Les Verts suisses (2001), «Non au blé transgénique de l'EPFZ. Pas d'expériences de génie génétiques risquées et inutiles», communiqué de presse du 12.1.2001, url: http://www. verts. $\mathrm{ch} / \mathrm{f} /$ politik/text.asp $? \mathrm{id}=28 \& \mathrm{t} \_$pid $=22 \& \mathrm{e}=2 \& \mathrm{~b}=1 \& \mathrm{ub}=11 \&$ uub $=32 \&$ page $=11$

25 Interview de Christophe Sautter, le 11.12.2001, IMédia -Observatoire EPFL, non publié.

26 CFSB, «Prise de position de la CFSB concernant la demande B00003 de l'Institut de biologie végétale de l'EPFZ, Comportement des variétés de blé transgénique KP4 en plein champs », 5 septembre 2001, url: http://www.umwelt-schweiz.ch/imperia/md/content/efbs/16.pdf, p. 11.

27 Ibid., p.10.

${ }_{28}$ Le service pour le paysage et la nature, le laboratoire cantonal, le service médical ainsi que le service vétérinaire cantonal.

$29 \mathrm{CENH}$, «Stellungnahme zum Gesuch des Institutes für Pflanzenwissenschaften des ETH Zürich um Bewilligung eines Freisetzungversuches mit gentechnisch verändertem KP4-Weizen in Eschikon ZH», 5.4.2001, url: http://www.umwelt-schweiz.ch/imperia/md/content/ekah/10.pdf 
l'évaluation des buts et des effets écologiques, économiques et sociaux de cette dissémination, ainsi que sur des questions de fond, la CENH est elle aussi restée dans le cadre de son référentiel: l'éthique. En ce qui concerne l'évaluation des buts de l'essai, la CENH met en évidence leur diversité mais également un manque de clarté dans leur exposition. Elle relativise en outre la contribution que peut apporter cette dissémination à une recherche en biosécurité ainsi que les réponses qu'elle pourrait apporter à des problèmes agronomiques. Dans son évaluation des effets, la CENH fait remarquer que, bien qu'à un stade très précoce, cette recherche est orientée vers une application concrète et que les effets possibles à long terme, y compris ceux résultant d'un emploi commercial, doivent donc être pris en compte ${ }^{30}$. Parmi les effets écologiques, la question de la résistance aux antibiotiques est abordée et, bien que sa dangerosité écologique soit écartée, elle est considérée comme problématique du fait de l'impact social (Signalwirkung) qu'elle peut avoir. La mention des risques résiduels de la part des requérants en ce qui concerne les mesures de sécurité sur le site de l'essai est saluée par la commission alors que les bénéfices découlant d'une éventuelle diminution de l'emploi de pesticide sont considérés comme difficiles à évaluer à ce stade d'avancement du projet. Enfin, l'absence de discussion des méthodes alternatives permettant de remplir les mêmes objectifs est critiquée. La CENH estime en effet qu'une telle mise en perspective devrait figurer dans toute demande de dissémination afin d'évaluer sa pertinence et sa capacité à apporter une solution à un problème réel. Les effets économiques sont également considérés comme difficilement évaluables en raison du caractère plutôt fondamental de la recherche. Si cet essai devait mener au développement d'une application commerciale, alors un bénéfice pourrait être attendu d'une diminution de l'emploi de fongicides, mais parallèlement une analyse détaillée des méthodes alternatives devrait être effectuée pour pouvoir juger de la pertinence de ce projet.

En plus de ces avis requis par l'ordonnance sur les disséminations expérimentales, l'OFEFP demanda à un expert interne d'évaluer la demande de l'EPFZ. Ce dernier en vint à la conclusion que, malgré les demandes d'informations complémentaires adressées au requérant, la demande reste lacunaire et que, si l'essai devait être autorisé, des mesures de sécurité supplémentaires devraient être appliquées.

C'est donc sur la base de ces diverses recommandations que l'OFEFP fut amené à se prononcer le 20 novembre 2001 sur cette demande de dissémination. La demande fut refusée. Les arguments de l'OFEFP étaient les suivants:

- La demande est lacunaire et ne contient pas les informations nécessaires concernant la caractérisation moléculaire de la plante (description de la séquence, nombre de copie de la séquence, site d'insertion et expression). Il en résulte que les dommages potentiels du blé KP4 ne peuvent être évalués.

- Le spectre d'action de la protéine KP4 est sujet à controverse et il pourrait affecter d'autres champignons.

30 Ibid, p. 3 . 
- Aucune étude sur les effets de la protéine KP4 sur des organismes utiles ou non ciblés n'a été effectuée; des dommages pour l'environnement ne peuvent donc pas être exclus.

- La présence d'un gène de résistance à l'antibiotique ampicilline, utilisé également en médecine humaine, peut indirectement affecter l'environnement et la santé humaine. Ce gène n'étant pas nécessaire dans la plante et résultant de l'usage d'une technologie dépassée, la demande ne peut être acceptée, en conformité avec le principe de précaution.

- Un croisement avec une autre plante de blé ou de seigle des champs environnants ne peut être exclue.

Bien qu'ayant abouti à une décision en contradiction avec tous les avis émis, à l'exception de son expert interne, l'autorité de régulation est restée dans le cadre d'une argumentation strictement scientifique relative aux risques pour la santé et l'environnement qui a été celle de l'ensemble de la procédure de consultation, à l'exception de la CENH. Interprétées différemment, ce sont les mêmes et identiques questions qui ont été traitées par l'OFEFP et par les instances consultées. Il est intéressant de noter qu'aucune des critiques formulées par la CENH n'a été utilisée pour justifier le refus. Ainsi, c'est peut-être à juste titre que, au début de sa prise de position, la CENH rappelle que, en raison de l'absence de bases légales nécessaires, les recommandations qu'elle émet ne peuvent pas, dans chaque cas, être prises en considération dans la décision rendue par l'autorité compétente. Ainsi, le point de vue de la CENH n'est pas seulement destiné à conseiller les autorités exécutives, mais également à émettre un avis sur les bases légales en vigueur $^{31}$. Parmi les arguments critiques à l'égard de l'essai figurait notamment le fait que l'utilisation des gènes de résistance aux antibiotiques posait un problème social, de par «l'effet d'annonce» engendré, indépendamment du risque pour la santé et l'environnement.

La décision de l'OFEFP suscita des réactions pour le moins contradictoires et marqua en réalité le début d'une bataille juridique haute en rebondissements qui allait s'engouffrer dans les méandres d'une législation qui se révéla encore mal adaptée à encadrer ce type de procédure. Saluée par les mouvement écologistes et en particulier par les Verts suisses ${ }^{32}$, qui s'étaient déjà exprimés précédemment contre cette dissémination $^{33}$, le refus de l'OFEFP suscita en revanche les critiques des partis bourgeois et la consternation parmi nombre de chercheurs. La polarisation politique entre la gauche, dans l'ensemble plutôt défavorable aux disséminations expérimentales, et la droite soutenant les OGM et la recherche, était très nette. Après les deux demandes de dissémination refusées par l'OFEFP en 1999, la décision concernant le blé de l'EPFZ constituait en quelque sorte un signal qui

${ }^{31} \quad$ Ibid. p. 1.

32 Les Verts suisses, «dissémination d'OGM: demande de l'EPFZ rejetée. Bravo l'OFEFP», communiqué de presse du 20.11.2001, url: http://www.verts.ch/f/politik/text.asp?id= $31 \& \mathrm{t} \_$pid $=25 \& \mathrm{e}=2 \& \mathrm{~b}=1 \& \mathrm{ub}=11 \& u u b=32 \&$ page $=7$

33 Les Verts Suisses, «Non au blé transgénique de l'EPFZ, pas d'expérimentation de génie génétique risquée et inutile», communiqué de presse du 12.01.2001, url: http://www.verts.ch/f/politik/ text.asp $? \mathrm{id}=28 \& \mathrm{t} \_$pid $=22 \& \mathrm{e}=2 \& \mathrm{~b}=1 \& \mathrm{ub}=11 \& \mathrm{uub}=32 \&$ page $=11$ 
allait permettre aux chercheurs actifs dans le domaine des plantes transgéniques, mais également à l'industrie de sonder le terrain. Or, avec cette décision, la possibilité d'effectuer des essais en plein champ se révélait plus éloignée que jamais ${ }^{34}$.

Sept jours après le refus de l'OFEFP, cinq membres de la CFSB annoncent leur démission. Dans un communiqué de presse, ils répondent ainsi à chacun des arguments de l'OFEFP et, bien qu'acceptant la décision comme telle, ils récusent les motifs sur lesquels se fonde son argumentation ${ }^{35}$. Le débat public s'intensifie. Deux arènes sont déjà impliquées suite à ce conflit entre autorité de régulation et experts, l'arène régulatrice par le biais de l'OFEFP et l'arène scientifique par le biais de la CFSB. Le débat se focalise immédiatement sur des arguments scientifiques complexes, pour lesquels les données expérimentales ne sont pas complètes; l'essentiel du débat a d'ailleurs lieu entre experts. Risques relatifs aux gènes de résistance à l'ampicilline, risque de dissémination de pollen, risques liés à une description moléculaire incomplète et aux effets de la protéine KP4, voilà l'essentiel des points controversés.

L'Institut de biologie végétale de l'EPFZ décide alors de faire recours auprès du DETEC contre le refus de l'OFEFP. Il conteste chacun des arguments justifiant le refus. A travers une argumentation complexe, il met en évidence que l'OFEFP aurait exigé des renseignements qui n'étaient pas nécessaires pour évaluer les risques. Par ailleurs, des interprétations scientifiquement inexactes auraient été effectuées, notamment en ce qui concerne le gène de résistance à l'ampicilline; le risque a en effet été évalué de manière abstraite et globale plutôt qu'en se basant sur les conditions précises de l'expérience. A ce sujet, l'interdiction d'application anticipée d'une loi encore en discussion au parlement aurait par ailleurs été enfreinte. Les exigences relatives à la caractérisation moléculaire auraient également été trop élevées en regard de la dimension de l'essai et de son caractère expérimental. En ce qui concerne les risques de la protéine KP4, l'OFEFP s'est basé sur une demande de brevet évoquant un large spectre d'action. Or, pour l'EPFZ, une demande de brevet ne peut pas être prise en considération puisque son but est avant tout économique et non pas scientifique. Contestant le dernier argument de l'OFEFP relatif aux risques de dissémination de pollen, les auteurs du recours indiquent que l'OFEFP s'est à nouveau basé sur une évaluation générale plutôt que sur les conditions concrètes de l'essai et que les éventuels préjudices résultant d'une dissémination de pollen seraient tellement minimes qu'ils ne peuvent être considérés comme un risque pour l'environnement au sens légal. Enfin, en ne tenant pas compte du fait que les conditions proposées par les différentes instances consultées auraient suffit, l'OFEFP aurait entravé la liberté de recherche. L'instance de décision se serait ainsi écartée des prises de position des commissions et des offices consultés sans argumenter et justifier ces divergences.

Dans sa décision du 12 septembre 2002, le DETEC accepte le recours de l'EPFZ. L'ensemble des points soulevés par l'EPFZ est accepté à l'exception de la question de la liberté de recherche, sur lequel le DETEC jugea inutile de se

${ }^{34}$ A noter que, suite à cette décision, la compétence de l'OFEFP en matière d'autorisation pour les disséminations expérimentales fut remise en question et, le 27 janvier 2003, le Conseil fédéral dut confirmer l'OFEFP dans ses fonctions. (Gen Suisse (27.1.2003) «Dissémination de plantes contenant des OGM: L'OFEFP reste l'organe compétent pour les autorisations »)

35 CFSB, communiqué de presse, 27 novembre 2001. 
prononcer. La demande de dissémination expérimentale est donc autorisée mais les conditions précises de son exécution doivent encore être fixées par l'OFEFP qui doit réévaluer la demande.

Ce recours déposé auprès de l'autorité de tutelle de l'OFEFP peut être assimilé à l'intrusion d'une troisième arène dans le débat, l'arène juridique. Toutefois, du fait de diverses similitudes et recoupements entre l'arène de régulation et l'arène juridique, il est difficile de voir à ce stade le développement d'une controverse publique $^{36}$. En effet, le débat reste pour l'essentiel cantonné au niveau des autorités en place, qu'elles soient scientifiques, administratives ou juridiques et il est essentiellement le fait des experts, juristes ou chercheurs.

C'est alors que la société civile, au premier rang de laquelle les riverains du site d'expérimentation, mais aussi les paysans suisses de production intégrée regroupés dans l'association IP-Suisse et l'association de défense de l'environnement Greenpeace sortent de leur réserve. Dans un communiqué daté du 13 septembre 2002, Greenpeace critique le département de Moritz Leuenberger et l'accuse d' "avoir fini par céder aux pressions du lobby de la transgenèse et accepté de négliger la protection de la santé publique et de l'environnement $»^{37}$. Elle argumente son opposition à la dissémination de blé transgénique de la manière suivante:

«Comme il est déjà possible de lutter contre la carie du blé avec des moyens éprouvés et ménageant l'environnement, l'expérimentation en plein champ demandée par l'EPFZ revient à prendre un risque inutile. Jusqu'à présent, l'agriculture suisse est protégée contre les plantes transgéniques. Cette situation n'est pas seulement nécessaire d'un point de vue environnemental mais aussi du point de vue économique car elle permet à l'agriculture suisse, qui mise sur la qualité, de se targuer d'une production garantie sans génie génétique. [...]

Nous utiliserons tous les moyens à notre disposition pour empêcher la dissémination de plantes transgéniques et protéger la population et son environnement de leurs effets imprévisibles. Les expériences doivent se faire en laboratoire, pas en plein champ. Un sondage que Greenpeace a mandaté en août montre que 73,3\% des Suissesses et des Suisses partagent cette opinion. [...] La carie du blé ne constitue pas un problème pour l'agriculture suisse; et lors des rares fois où elle se manifeste, il est possible de lutter contre elle avec des moyens plus fins que la massue du génie génétique [...] La décision du DETEC est un camouflet à la population suisse et à l'OFEFP et crée [...] un précédent qui va à l'encontre du projet de loi proposé au parlement. Les faits continuent en outre à s'opposer à une autorisation; la demande présentée n'est pas suffisante. Pour des essais de dissémination, il faut en effet au moins que les organismes soient complètement caractérisés et accompagnés d'une étude optimales des risques.» ${ }^{38}$

\footnotetext{
${ }^{36}$ Rappelons que, selon la définition de Joly, un débat ne devient une controverse publique que lorsqu'il mobilise plus de deux arènes et qu'il y a un nombre croissant d'interactions entre arènes (Joly et al., 2001, op. cit.).

37 Greenpeace Suisse, «Greenpeace annonce la résistance à la décision du Conseil fédéral concernant le blé transgénique», 13.09.2002, url: info.greenpeace.ch/fr/genie/Pressreleases/ cp_130902_ble

38 Ibid.
} 
La mention par Greenpeace des risques pour l'agriculture suisse, de la pertinence de l'utilisation du génie génétique dans le cas d'une maladie qui peut être combattue par d'autres moyens, de l'agenda politique et des lois en discussion au parlement marquent l'entrée en scène d'un nouveau cadre référentiel, le contexte socio-économique dans lequel devrait avoir lieu cet essai. Toutefois, Greenpeace ne se limite pas à élargir le débat et reprend à son compte divers arguments de type scientifique concernant les risques pour la santé et l'environnement comme la question du gène de résistance à l'ampicilline, les effets imprévisibles des plantes transgéniques sur la population et l'environnement ainsi que la caractérisation moléculaire insuffisante.

Il est intéressant de noter que la plupart des arguments de type socio-économique évoqués ici par Greenpeace avaient déjà été soulevés par la CFSB. Dans une section figurant tout à la fin de sa prise de position, après la conclusion, la CFSB a jugé bon de mentionner des «Points soulevés au cours de la discussion », indépendamment de l'aspect de sécurité biologique. Ces points, de nature plutôt critique par rapport à la demande de dissémination, étaient les suivants :

«- La culture de blé conventionnel n'est pas d'une importance majeure en Suisse, la demande intérieure en blé IP et bio étant en constante augmentation [...]

- L'approche globale prise en choisissant le gène $k p 4$ est jugée très compliquée et coûteuse [...]

- Les méthodes de désinfection utilisées à travers le monde pour la culture de blé biologique donnent en outre de très bons résultats [...]

- Par ailleurs, l'éventuelle contribution à la promotion de l'agriculture biologique, à la réduction de la quantité de pesticides utilisés [...] et à l'amélioration de l'approvisionnement mondial en denrées alimentaires, mentionnée à plusieurs reprises à propos de la présente dissémination expérimentale, est mise en doute, aucune preuve scientifique ne pouvant étayer à l'heure actuelle de telles affirmations ».

Ainsi, face à cette demande, la CFSB avait jugé bon de se prononcer également sur des aspects ne relevant pas directement de son mandat et prenant en considération des aspects allant au-delà de la question de la biosécurité, notamment la situation spécifique de l'agriculture suisse, les techniques alternatives disponibles ainsi que l'utilité de cette plante transgénique en regard d'objectifs généraux tels que la promotion de l'agriculture biologique, une diminution de l'emploi de pesticides et la sécurité alimentaire au plan mondial. Ces arguments étant clairement un ajout en regard du mandat de la CFSB - ils ont été relégués après la conclusion par la CFSB elle-même - aucun ne fut pris en compte dans la décision de l'OFEFP.

Les diverses associations et mouvements mobilisés contre cette dissémination décident toutefois d'attendre la décision de l'OFEFP pour recourir. Cette dernière tombe le 20 décembre 2002 et autorise la dissémination expérimentale de l'EPFZ, sous certaines conditions. Le même jour, des militants de Greenpeace escaladent les bâtiments de l'EPFZ en signe de protestation contre la décision de l'OFEFP. Dans un communiqué de Greenpeace, une habitante de Lindau témoigne: 
«Il y a une année, nous nous sommes réjouis du refus de l'OFEFP, soutenable et favorable à la santé publique et à l'environnement. Aujourd'hui, toutes les motivations de ce refus sont interprétées différemment et/ou pas prises en considération. Nous ne le comprenons pas et allons nous y opposer.» ${ }^{39}$

L'action directe de Greenpeace contre l'EPFZ signifie l'entrée en scène d'une nouvelle arène dans le débat public, l'arène politique (les associations), et par conséquent l'intensification du débat qu'on peut dès lors qualifier de controverse publique. On assiste alors à deux types de débordement: celui de l'arène scientifique sur celle de régulation (la démission des experts) et celui de l'arène politique sur l'arène scientifique (l'action directe de Greenpeace contre l'EPFZ).

Outre divers arguments déjà évoqués dans son précédent communiqué sur ce dossier, Greenpeace relève que les consommateurs ne veulent pas d'aliments transgéniques et que les informations concernant les effets environnementaux négatifs des plantes transgéniques aux Etats-Unis s'accumulent, rendant cet essai inadmissible. Enfin, toujours selon Greenpeace, le fait que le blé transgénique de l'EPFZ planté sous serre en 2001 n'ait pas présenté la résistance escomptée rend la poursuite de l'essai en plein champ encore plus absurde. Débute alors une intense mobilisation des opposants à l'essai.

Le 30 janvier 2003, IP-Suisse fait recours auprès du DETEC contre l'autorisation accordée par l'OFEFP. Les riverains du site organisé en groupe de travail «Lindau contre le blé transgénique», Greenpeace Suisse, les Verts suisses, KAGfreiland $^{40}$, Swissaid, l'association des petits et moyens agriculteurs VKMB, Pro Natura ainsi que l'association des producteurs bio zurichois et schaffousois s'associent au recours.

Ainsi, selon les recourants:

«les documents fourni par l'EPFZ concernant l'essai prévu sont remplis de questions sans réponses et de contradictions. Des risques pour les humains, l'environnement et l'agriculture restent possibles lors de cette expérience insensée d'un point de vue écologique et agricole dont le risque n'a en outre pas été analysé comme le veut l'Ordonnance fédérale sur la dissémination dans l'environnement $(\mathrm{ODE}) . »^{41}$

Selon Bruno Heizer, le responsable de la campagne génie génétique de Greenpeace:

«Toutes les raisons du refus de l'OFEFP en 2001 restent d'actualité: antibiorésistance, menace pour les insectes, les bactéries du sol, transfert de gènes vers les blés et les graminées.»

Il souligne en outre l'ignorance des conséquences d'une intervention dans le patrimoine héréditaire ainsi que la méconnaissance des interactions entre les organismes et leurs biotopes ${ }^{42}$.

\footnotetext{
39 Greenpeace, «Des habitants de Lindau et Greenpeace font recours contre la décision de disséminer du blé transgénique à Lindau», communiqué de presse du 20.12.2002, url: http://info.greenpeace.ch/fr/genie/communiques/cp_201202_epfz

40 Konsument/innen-Arbeitsgruppe für tier- und umweltfreudliche Nutztierhaltung.

${ }^{41}$ Greenpeace, «Des riverains, des agriculteurs PI et Greenpeace recourent contre le blé transgénique», communiqué de presse du 30.1.2003, url: http://info.greenpeace.ch/fr/genie/communiques/cp_300103_epfz

42 Ibid.
} 
Face à cette dernière argumentation, une remarque s'impose. En effet, alors qu'ils avaient élargi le champ des paramètres pertinents pour l'évaluation de la demande de dissémination, lorsqu'il s'agit de justifier le recours face aux autorités, les opposants à l'essai restreignent drastiquement leur argumentation, ne faisant état que de questions scientifiques et juridiques. Ainsi, il est intéressant de noter que la mobilisation de l'arène juridique de la part des associations tend à leur faire délaisser le référentiel propre à leur «arène d'origine», l'arène politique. L'impact de cette stratégie sur l'évolution du débat sera analysé de manière plus détaillée dans la section suivante.

Le DETEC décide toutefois de lever l'effet suspensif du recours ${ }^{43}$. Il invoque pour cela le fait que l'expérimentation de l'EPFZ risque d'être définitivement compromise si elle ne peut effectuer son essai en 2003 car son financement touche à sa fin. Par ailleurs, les recourant ne s'étant pas manifestés lors de l'acceptation du recours de l'EPFZ, ils avaient de fait accepté le principe de l'essai.

Le 10 mars 2003, les opposants à l'essai déposent un recours auprès du Tribunal fédéral contre la levée de l'effet suspensif du recours décidé par le DETEC. Sans l'effet suspensif, l'essai pouvait en effet se dérouler sans que l'affaire ait encore été jugée sur le fond des arguments présentés par les recourrant. Dans un arrêté du 13 mars 2003, le Tribunal fédéral jugea que le DETEC avait estimé à tort que les opposants avaient déjà eu la possibilité de faire recours contre sa décision du 12 septembre 2002 d'une part, et que toute possibilité d'obtenir une prolongation du financement du projet de l'EPFZ était exclue d'autre part. Il décida donc de rétablir l'effet suspensif du recours de IP-Suisse et des autres associations. Les opposants à l'essai poursuivent leur stratégie qui consiste à mobiliser en premier lieu l'arène juridique.

La dissémination expérimentale de l'EPFZ n'allait donc pas pouvoir avoir lieu avant que le DETEC ne se soit prononcé sur le fond des arguments avancés par les opposants à l'essai, ce qui concrètement allait reporter l'expérience d'une année au minimum.

En avril 2003, l'EPFZ demanda à ce que l'ensemble de la procédure d'autorisation soit repris depuis le début, requête qui fut soutenue par les opposants ayant fait recours contre la décision de l'OFEFP du 20 décembre 2002. Le 13 juin 2003, le DETEC accepta le recours des opposants à la dissémination et décréta que la procédure devait être reprise à zéro. Le DETEC n'analysa donc pas le fond des arguments invoqués par les opposants à cette dissémination, mais n'eut à se prononcer que d'un point de vue formel sur l'acceptabilité juridique d'une reprise de l'ensemble de la procédure d'autorisation. Une nouvelle demande de dissémination fut donc publiée le 22 juillet 2003 dans la Feuille fédérale. Contrairement à la première demande de dissémination pour laquelle aucune précision n'avait été stipulée concernant les modalités de recours, toutes les personnes désirant faire valoir leurs droits comme partie à la procédure devront le faire savoir dans un délai de 60 jours à compter de la date de publication ${ }^{44}$.

43 DETEC, «La dissémination expérimentale de l'EPFZ pourrait commencer ce printemps » communiqué de presse du 21 février 2003, url: http://www.uvek.admin.ch/dokumentation/ medienmitteilungen/artikel/20030221/01303/index.html ?lang $=\mathrm{fr}$

44 Une demande de l'EPFZ pour réduire ce délai de 90 à 60 jours avait été acceptée. 
Le 30 octobre 2003, l'OFEFP autorise une nouvelle foi la demande de l'EPFZ $^{45}$. Les instances consultées sont les mêmes que lors de la première demande. Toutes émettent un avis favorable, à l'exception de la CENH qui émet des doutes concernant les buts scientifiques de la tentative et recommande de ce fait de ne pas autoriser l'essai tant que cet aspect n'est pas éclairci ${ }^{46}$. La CENH précise en outre que les bénéfices d'une dissémination devraient également être pris en compte. Le 27 novembre 2003, Greenpeace et IP Suisse font recours auprès du DETEC contre l'autorisation de l'OFEFP. Ce recours est rejeté le 27 février 2004 et le blé transgénique est finalement planté sur la commune de Lindau le 18 mars 2004.

\section{ANALYSE ET COMPARAISON DU DÉBAT PUBLIC EN FRANCE ET EN SUISSE}

Les débats publics suisse et français concernant les disséminations expérimentales ont évolué de manière souvent différente, mais ont également présenté un certain nombre de similitudes. Avant de rentrer dans le détail de la comparaison entre ces deux pays, un certain nombre d'éléments généraux doivent être mentionnés.

En premier lieu, il est important de relever que la France a une organisation politique très centralisée, alors que la Suisse est au contraire organisée selon un système fédéraliste et décentralisé, qui prévoit la possibilité d'une participation importante des citoyens à la vie politique à travers son système de démocratie directe, dont l'un des principaux instruments est l'initiative populaire. C'est d'ailleurs par ce biais que le débat concernant les OGM a été lancé en Suisse en 1993 avec l'initiative «Pour la protection génétique». En France en revanche, le débat n'a réellement débuté qu'en 1996, à travers les actions directes de Greenpeace pour bloquer l'arrivage sur sol français de cargos transportant des OGM.

En ce qui concerne la recherche sur les OGM, alors que la France dispose d'une recherche publique bien implantée et soutenue tant financièrement que politiquement - notamment à travers les centres de l'INRA ${ }^{47}$ - les moyens de la recherche publique sur les OGM en Suisse sont en comparaison dérisoires, avec tout au plus deux institutions travaillant au développement d'OGM susceptibles d'être commercialisés un jour ${ }^{48}$.

45 OFEFP, «Verfügung vom 30. Oktober 2003, Referenz-Nr. B00003», url: http://www.umweltschweiz.ch/imperia/mdcontent/buwalcontent/folder/03-10-30eth/1.pdf

46 CENH, «B00003: Stellungnahme zum aktualisierten Gesuch für einen Freisetzungsversuch mit transgenen KP4-Weizen-Varietäten des Institutes für Pflanzenwissenschaften der ETH Zürich vom 26. Juni $2003 », 10$ septembre 2003, url: http://www.umwelt-schweiz.ch/imperia/md/ content/ekah/71.pdf

47 Institut national de recherche agronomique.

48 Il s'agit de la Station fédérale de recherche en production végétale de Changins et de l'EPFZ. A noter que d'autres institutions travaillent avec des plantes transgéniques, mais leur propos est généralement de faire de la recherche fondamentale ou alors de la recherche sur les risques (Station fédérale de Reckenholz) et non pas le développement d'une plante transgénique destinée à l'agriculture. 
Enfin, le dernier élément a trait à la quantité d'OGM plantés. Comme nous l'avons mentionné, les seuls OGM jamais plantés en Suisse l'ont été en 19911992 dans un cadre expérimental. Depuis lors aucun OGM n'a été cultivé en Suisse. La France a en revanche été un pays pionnier en la matière. En plus des disséminations expérimentales, environ une trentaine en 2003, la France a également des cultures commerciales de maïs OGM. A noter toutefois qu'en 1998 la surface totale de plantes transgéniques avoisinait les 1'500 ha, alors quelle est à ce jour inférieure à 100 hectares.

\subsection{Cadre légal}

En Suisse, le cadre légal qui a régi la prise de décision en matière de disséminations expérimentales d'OGM jusqu'à fin 2003 est la loi fédérale sur la protection de l'environnement (LPE) ${ }^{49}$ accompagnée de l'une de ses ordonnances, celle sur l'utilisation d'organismes dans l'environnement (ODE). Cette législation a toutefois été remplacée, puisqu'une loi sectorielle traitant spécifiquement des questions relatives au génie génétique dans le domaine non humain (LGG) a été adoptée par le parlement en mars 2003 et est entrée en vigueur en début 2004. Nous reviendrons plus tard sur cette loi, mais il convient ici de souligner que toute la controverse sur le blé KP4 a eu lieu sous la LPE et c'est donc de cette dernière et de son ordonnance d'application dont nous allons traiter principalement.

Comme déjà mentionné, le but de la LPE est de protéger les hommes et l'environnement des atteintes nuisibles ${ }^{50}$. Les dispositions spécifiques de la LPE relatives aux organismes dangereux pour l'environnement ainsi que l'ODE visent naturellement les mêmes objectifs ${ }^{51}$. Les disséminations expérimentales sont régies par les articles 7 à 12 ODE et l'article 8 ODE précise les conditions d'autorisation $^{52}$. Enfin, en conformité avec l'annexe 4 de l'ODE, les risques doivent être évalué de manière scientifique ${ }^{53}$. Les disséminations expérimentales sont donc réglementées de telle sorte à ce que les risques pour l'homme et pour l'environnement soient évités. La loi ne mentionne aucun autre paramètre en fonction duquel évaluer les demandes de disséminations expérimentales.

Etat du 27 novembre 2001.

so La présente loi a pour but de protéger les hommes, les animaux, les plantes, leurs biocénoses et leurs biotopes des atteintes nuisibles ou incommodantes, et de conserver la fertilité du sol (art.1 LPE).

51 Voir art. 29 LPE et art. 1 ODE.

52 Les disséminations expérimentales sont autorisées si l'on peut exclure qu'elles puissent:

- porter atteinte à la population d'organismes protégés ou importants pour l'écosystème touché;

- entraîner la disparition d'une quelconque espèce d'organismes ;

- perturber de manière importante ou durable l'équilibre des composants de l'environnement;

- porter atteinte de manière importante ou durable à des fonctions importantes de l'écosystème touché, en particulier à la fertilité du sol;

- entraîner la propagation permanente de propriétés indésirables dans d'autres organismes. «L'évaluation du risque doit être scientifique et reproductible, et se fonder sur des données scientifiques et techniques issues de l'expérience, des publications spécialisées, des résultats de calculs et des analyses détaillées ». Remarque, annexe 4, ODE. 
Durant la procédure de consultation qui précède toute décision concernant une demande de dissémination expérimentale, les instances amenées à émettre un avis ont donc dû le faire en se basant sur cette législation. Pour la CFSB, cela n'a nullement posé problème puisque son mandat est précisément celui d'évaluer les risques sanitaires et environnementaux relatifs aux OGM. Quant aux offices fédéraux (OFAG, OVF, OFSP) et à l'AWEL, ils ont évalué les risques pour la santé et l'environnement en fonction de leur domaine de compétence spécifique. En revanche, la CENH s'est retrouvée dans une position pour le moins singulière. Conformément à son mandat, la CENH a effectué une évaluation de la demande du point de vue éthique. Or, faute de base légale, son avis ne peut être pris en compte dans la décision. Pour pallier ce hiatus, dont la commission d'éthique semble bien consciente, une modalité de consultation que l'on pourrait qualifier d'indirecte a été mise en place. Ainsi, lorsqu'elle se prononce sur un cas particulier de dissémination expérimentale, ses remarques doivent davantage être vues comme des propositions d'interprétation de la législation en cours d'élaboration (LGG en préparation) que comme un avis sur la décision à prendre ${ }^{54}$.

Le cadre légal en vigueur établit donc de facto une distinction majeure entre les arguments scientifiques d'une part et les arguments socio-économiques, éthiques et politiques d'autre part. Les premiers sont ainsi les seuls sur lesquels la décision d'autorisation puisse se fonder. On comprend dès lors mieux pourquoi toute l'argumentation des instances consultées faisait uniquement état d'arguments de type scientifique, excepté la CENH qui toutefois soulignait que son avis ne saurait dans chaque cas être pris en compte dans la décision. Mais au-delà des instances consultées, on peut d'ores et déjà percevoir que ce cadre légal est également susceptible d'influencer le discours d'autres acteurs de la controverse, en particulier les opposants à la dissémination qui, dans la mesure où ils tentent de convaincre les instances officielles, auront tout avantage à utiliser des arguments «valables» du point de vue légal. Ce très bref examen de la législation suisse met donc en évidence des contraintes importantes, de nature à influencer le débat public. Est-ce à dire alors que la France, qui a connu un autre type de débat public, dispose d'une législation substantiellement différente?

En France, deux niveaux législatifs sont pertinents pour ce qui est des disséminations d'OGM: le droit communautaire d'une part, et le droit national d'autre part. Au niveau du droit communautaire, la directive 2001/18/CE du 12 mars 2001 réglemente la dissémination volontaire d'organismes génétiquement modifiés dans l'environnement. Elle a été traduite en droit national français dans le code de l'environnement ${ }^{55}$. Le droit communautaire étant fort complexe et notre propos n'étant pas ici de faire une étude de droit comparé, notre analyse se bornera à déterminer si la législation applicable en France permet de justifier une décision sur des arguments autres que scientifiques.

54 Voir CENH, «Stellungnahme zum Gesuch des Institutes für Pflanzenwissenschaften des ETH Zürich um Bewilligung eines Freisetzungversuches mit gentechnisch verändertem KP4-Weizen in Eschikon ZH», 5.4.2001, url: http://www.umwelt-schweiz.ch/imperia/md/content/ekah/ 10.pdf, p.1.

s5 Dispositions générales: art. L531. Utilisation confinée des organismes génétiquement modifies: art. L.532. Dissémination volontaire et mise sur le marché d'organismes génétiquement modifies: art. L.533. 
Tout comme la LPE en Suisse, la Directive 2001/18/CE a pour but de protéger la santé humaine et l'environnement, conformément au principe de précaution (art.1). Les disséminations expérimentales ${ }^{56}$ de même que la mise sur le marché sont soumises à autorisation et une évaluation des risques pour l'environnement est requise, conformément à l'annexe II de la directive. Mais au-delà de ces aspects similaires à la législation suisse, l'un des considérants de la directive introduit en particulier un élément intéressant. Le considérant 9 précise en effet que:

«Le respect des principes éthiques reconnus dans un Etat membre est particulièrement important. Les Etats membres peuvent prendre en considération des aspects éthiques lorsque des OGM sont volontairement disséminés ou mis sur le marché en tant que produits ou éléments de produits. $\gg^{57}$

Cet élément nous permet d'ores et déjà de dire que la directive européenne 2001/18/CE donne théoriquement aux autorités compétentes plus de latitude dans leurs décisions relatives aux disséminations expérimentales ainsi qu'à la mise sur le marché d'OGM que ne le permet la LPE en Suisse.

\subsection{Relation entre expertise et décision}

Mais revenons à la Suisse afin d'examiner un autre aspect de la question. Lorsqu'il a rendu ses décisions, l'OFEFP a toujours été parfaitement conscient des contraintes légales. Il ne s'y est d'ailleurs pas trompé puisqu'il a toujours justifié ses décisions par des arguments scientifiques. En inscrivant son argumentaire dans un cadre de référence scientifique, l'autorité de régulation n'a pas outrepassé les limites assignées par son arène. Rappelons que s'il a pu refuser la dissémination du blé KP4 malgré l'avis favorable des experts, et cela sans sortir du cadre scientifique, c'est parce qu'il a utilisé ces mêmes arguments tout en les interprétant différemment. Il a donc agi en conformité avec la loi. Pourquoi, dans ce cas, sa première décision a-t-elle été annulée par le DETEC en première instance? C'est parce que, selon le DETEC, l'interprétation des données scientifiques et l'évaluation de leur pertinence pour établir le degré de risque pour l'homme et l'environnement reviennent en premier lieu à la CFSB, à savoir aux experts scientifiques. Le DETEC précise en effet dans sa décision du 12 septembre 2002 que l'OFEFP n'est pas autorisé à s'écarter de la position de la CFSB sans raison valable.

En clair, au-delà de l'exigence légale de recourir uniquement à des arguments scientifiques relatifs aux risques environnementaux et sanitaires, la jurisprudence a également institué une autorité chargée de définir et d'interpréter de manière normative les données scientifiques disponibles en matière de risques pour la santé et pour l'environnement. Certes, il s'agit là d'une disposition tout à fait sage: à qui d'autre sinon aux experts scientifiques aurait-on pu confier un tel mandat? Les compétences des membres de la CFSB ne sauraient être remises en question. Pourtant, cela pose la question plus large de la relation entre deux étapes

\footnotetext{
56 Partie B: Dissémination volontaire d'OGM à toute autre fin que leur mise sur le marché.

57 Considérant 9, Directive 2001/18/CE
} 
que l'on conçoit généralement - et de manière très théorique ${ }^{58}$ - comme séparées : l'évaluation des risques qui relève de l'expertise et la gestion des risques qui relève du pouvoir politique (ou administratif). Si l'évaluation doit assurer à la décision un fondement scientifique aussi rigoureux que possible, la gestion des risques consiste à décider de l'adoption d'une mesure et de sa conception. L'enjeu de cette séparation est donc double: assurer à la décision un fondement scientifique aussi rigoureux que possible tout en reconnaissant une marge d'autonomie à l'autorité chargée in fine d'adopter une décision sur le risque (Noiville et de Sadeleer, 2001, p.393).

Aujourd'hui, le mandat des diverses instances émettant un avis préalablement à toute décision de dissémination expérimentale est sous-tendu par une législation et une jurisprudence qui fondent des distinctions majeures entre ces diverses instances. Bien qu'elles soient théoriquement toutes consultatives, et donc placées sur un pied d'égalité par rapport à la décision ${ }^{59}$, la CFSB a été investie d'une autorité qui dépasse de loin une fonction purement consultative: non seulement la décision du DETEC précise que l'évaluation des risques environnementaux et sanitaires est la prérogative de la CFSB, mais encore la législation en vigueur stipule que seuls ces risques permettent de justifier le refus d'une demande de dissémination expérimentale. On peut dès lors se demander s'il existe une distinction suffisante entre le rôle de la CFSB et celui de l'OFEFP, en d'autres termes entre l'instance d'expertise chargée de l'évaluation des risques et celle de décision en charge de la gestion des risques. La situation actuelle tendrait à suggérer que non, une bonne partie de la prérogative de décision s'étant en réalité transférée de l'OFEFP vers la CFSB où elle se superpose désormais à celle de l'expertise.

Mais au-delà du rôle de l'expertise scientifique et des contraintes légales, un autre problème demeure. Peut-être plus grave car moins évident et plus difficile à résoudre, il tend à escamoter le débat concernant les risques socio-économiques, éthiques et politiques des OGM et ce même parmi les opposants aux disséminations. Il s'agit de l'incapacité de l'arène politique à donner un certain degré de validité ou de vérité aux arguments qui sont développés en son sein, les rendant du même coup inutilisables pour fonder une quelconque décision de la part des autorités ${ }^{60}$. Pour les

${ }_{58}$ En effet, cette séparation ignore le fait que l'évaluation et la gestion des risques, loin de se suivre linéairement, se confondent souvent dans la pratique. En cas de crise par exemple, le temps manque pour effectuer une évaluation précise des risques avant d'adopter les mesures qui s'imposent. Mais cette séparation sous-tend également qu'il est possible de tracer une ligne de démarcation entre les faits et les valeurs, l'évaluation reposant sur des faits objectifs alors que la gestion des risques relèverait de la sphère des valeurs. Une telle dichotomie est réductrice, car l'évaluation est perméable aux jugements de valeurs, à plus forte raison lorsqu'elle doit faire face à des situations d'incertitude qui font appel à un raisonnement hypothétique. Voir dans ce même ouvrage le texte de Mark Hunyadi, «La logique du raisonnement de précaution».

59 Le processus de consultation est réglé par l'art. 18.4. ODE. A noter toutefois que bien que consultatifs, les offices fédéraux (OFAG, OVF et OFSP) ont en quelque sorte un «droit de veto » puisqu'une autorisation ne peut être délivrée que s'ils ont émis un préavis positif (art. 19.1.b. ODE).

${ }_{60}$ Cette problématique est développée ailleurs dans cet ouvrage sur la base de la distinction entre les menaces - qui sont des risques qui ne peuvent être validés - et les risques avérés ou hypothétiques qui peuvent être validés et qui correspondent en pratiques aux risques environnementaux et sanitaires. Voir: Bordogna Petriccione B. «De l'utilisation de la notion de risque dans le débat public sur les biotechnologies ». 
risques environnementaux et sanitaires, nous avons vu que la CFSB remplit cette fonction. Or, pour les autres types de risques, il n'existe rien de tel. Bien que la CENH ait été chargée de donner son avis d'un point de vue éthique sur les disséminations, ses positions ne constituent pas une référence obligatoire; elles ont de fait été en grande partie ignorées. Les effets socio-économiques et politiques quant à eux ne sont tout simplement pas évalués en tant que tels et seule la CENH en tient compte, mais en les évaluant d'un point de vue éthique uniquement. Il n'est évidemment pas question ici de regretter l'absence d'une structure autoritaire chargée d'établir la «vérité » dans ces domaines. La tyrannie n'étant pas notre propos, ce manque appelle au contraire la mise en place de procédures participatives couplées à des programmes de recherche spécifiques.

Mais ce n'est pas tout. D'autres éléments d'explication concernant la différence entre le débat en Suisse et le débat français méritent encore d'être examinés. A ce titre, il convient de mentionner l'organisation administrative et politique et en particulier la répartition des tâches de décision et d'expertise entre les structures strictement administratives et le gouvernement. Comme déjà mentionné, la législation et la jurisprudence suisse, à travers la décision du DETEC du 12 septembre 2002, ont donné un rôle prépondérant à la Commission fédérale d'experts pour la sécurité biologique (CFSB) dans les décisions concernant les demandes de dissémination expérimentales. Mais outre la législation et la jurisprudence, un autre aspect a également contribué à ce que l'on pourrait qualifier de glissement d'une partie du pouvoir décisionnel vers une instance qui devrait théoriquement n'être que consultative. Il s'agit du statut spécifique de l'OFEFP, l'instance chargée de la gestion des risques et, à ce titre, formellement investie du pouvoir de décision en matière de disséminations. Or, l'OFEFP est une structure qui fait partie de l'administration fédérale. Par conséquent, elle est hiérarchiquement subordonnée à son ministère de tutelle, le DETEC, et ne dispose pas d'une marge de manœuvre politique comparable à celle du gouvernement ${ }^{61}$.

La situation en France est quelque peu différente. Bien qu'elle ait dû faire face à une situation en tout point analogue à celle de la démission des experts de la CFSB en novembre 2001, elle y trouva une toute autre issue. En février 1997, suite à la décision du Premier ministre de l'époque, Alain Juppé, de retirer l'autorisation de cultiver le maïs Bt de Novartis, qui avait été accordée en décembre 1996, Axel Kahn, le président de la Commission du génie biomoléculaire (CGB) décida de démissionner. La CGB étant l'équivalent en France de la CFSB, les deux pays ont donc dû faire face à une démission de leurs experts suite à la non prise en compte de leur position. En France, pendant de nombreuses années, la concordance entre l'avis des experts et la décision des politiques avait été telle qu'il était devenu difficile de dire si cette situation était la conséquence d'une relation de dépendance des politiques face aux experts ou alors si experts et politiques s'étaient fortuitement retrouvés d'accord sur tout. La réponse arriva brutalement et provoqua une crise de légitimité au sein de la CGB qui estima que ses compétences étaient mises en doute. Toutefois, contrairement à la Suisse, les politiques

Cette subordination s'explique par le fait que le gouvernement est responsable des activités de l'administration devant le parlement (Pierre Moor, Droit administratif: les fondements généraux, vol. 1, éd. Staempfli, Berne, 1988, p. 12). 
français profitèrent de l'occasion pour reprendre leur autonomie. La Ministre de l'environnement commenta en effet la démission d'Axel Kahn de la manière suivante: «Cela signifiait que l'expert prend les décisions à la place du politique. Et bien non, ce n'est pas comme cela que ça fonctionne.» ${ }^{62}$ Chaque chose à sa place: l'expert expertise, le politique décide. Cette prise de distance de la part de l'autorité de gestion des risques par rapport à celle d'évaluation a probablement été rendue possible par le fait que, contrairement à la Suisse, la compétence pour octroyer des autorisations de dissémination ou de commercialisation ne revient pas à une instance administrative, mais directement au gouvernement en la personne du Ministre de l'agriculture, de l'alimentation, de la pêche et des affaires rurales, avec l'accord du Ministre de l'écologie et du développement durable.

Nous pouvons dès lors faire l'hypothèse qu'en Suisse le statut de l'OFEFP est l'un des facteurs qui tend à restreindre l'autonomie de l'administration en tant qu'autorité chargée de la gestion des risques par rapport à l'évaluation faite par les experts de la CFSB. Or, comme nous l'avons vu, les résultats de l'évaluation constituent une base nécessaire à la décision, mais ne devraient pas pour autant épuiser cet exercice politique (Noiville et de Sadeleer, 2001, p. 413). En France, en revanche, l'organisation administrative et politique semble permettre plus facilement une distinction entre le rôle des experts et celui des décideurs.

Avant de conclure sur cette question, il convient de relever quelques aspects historiques qui ont marqué différemment la relation entre la sphère de l'expertise et celle de la décision en France et en Suisse. La période ayant précédé la démission du comité d'expert (CFSB) en Suisse a été placée sous le signe de la discontinuité: par trois fois ${ }^{63}$, alors que la CFSB avait émis un avis favorable, l'OFEFP refusa la demande. Ce n'est qu'après ce que les membres de la CFSB ont considéré être un troisième «désaveu» qu'une partie d'entre eux décida de démissionner. En France en revanche, la décision Juppé faisait suite à près de dix ans de concordance parfaite entre les avis des experts de la CGB et les décisions du gouvernement. La démission d'Axel Kahn, alors président de la CGB, est donc intervenue dès la première prise de distance du gouvernement par rapport aux experts. Cette mise en perspective historique tend ainsi à suggérer que, bien qu'ayant été remis en question par la décision Juppé, l'impact de l'expertise sur la sphère de décision a été très significatif en France alors qu'il ne semble pas avoir été déterminant en Suisse, du moins jusqu'à la décision du DETEC du 12 septembre 2002 .

\subsection{Stratégie des opposants}

Le dernier élément permettant d'expliquer la divergence du débat en France et en Suisse concerne directement la stratégie des opposants aux disséminations. En Suisse, durant la période qui sépare la décision du DETEC du 12 septembre 2002 d'invalider le refus de la demande de dissémination du blé KP4 - décision prise par l'OFEFP en novembre 2001 - et le moment où les opposants font recours

62 Cité par Hervé Kempf, op.cit., p. 143.

63 Il s'agit des trois demandes déposées en Suisse à ce jour: celle concernant le mais résistant à un herbicide, celle concernant la pomme de terre résistante au mildiou et celle concernant le blé résistant à la carie. 
contre la nouvelle décision de l'OFEFP autorisant cette fois la dissémination, on assiste à un très net élargissement du débat, qui a été également l'occasion de diverses «démonstrations », telles que la mise en place d'un camp de tipi à proximité du site d'expérimentation ${ }^{64}$ et le dépôt d'un tas de fumier sur le lieu même de l'essai ${ }^{65}$.

Parmi les arguments invoqués, on trouve notamment:

- la question de l'utilité ou plutôt de l'inutilité agronomique de cet essai (la procédure d'évaluation n'examine actuellement que les risques);

- la nécessité de protéger l'agriculture suisse pour des raisons aussi bien environnementales qu'économiques (l'idée étant que si l'agriculture suisse veut miser sur la qualité, elle ne doit pas produire d'OGM);

- le fait que l'opinion publique est défavorable aux OGM;

- et qu'une loi (la LGG) prévoyant des conditions d'autorisation plus stricte que la législation en vigueur est en préparation au parlement ${ }^{66}$.

Ces éléments méritaient sans aucun doute d'être débattus, puisqu'ils posent des questions de fond qui en réalité sous-tendent tout le débat sur les OGM. Or, nous l'avons vu, les opposants aux disséminations ont subitement restreint leur discours. Ayant choisi d'attaquer la décision de l'OFEFP du 20 décembre 2002 autorisant la dissémination par voie judiciaire en faisant recours auprès du DETEC puis auprès du Tribunal fédéral, les opposants ont adopté une stratégie judiciaire. Ce faisant, ils ont de facto accepté l'autorité du pouvoir judiciaire et la pertinence du cadre législatif en vigueur pour déterminer les catégories d'arguments pertinents pour l'évaluation d'une demande de dissémination, en l'occurrence les arguments scientifiques et juridiques. En d'autres termes, ils se sont pliés au mode de fonctionnement de l'arène qu'ils contestaient (celle de l'autorité de régulation), ce qui les a empêchés d'imposer leur propre cadre de référence.

En France, la stratégie des opposants a varié d'un acteur à l'autre. Comme en Suisse, Greenpeace a agi sur deux fronts, celui des actions directes, comme nous l'avons vu dans la première partie, et celui des actions judiciaires. Elle est en effet à l'origine d'un recours auprès du Conseil d'Etat, l'une des plus hautes autorités légales françaises, contre la décision du 5 février 1998 autorisant la mise en culture du maïs Bt 176 de Novartis. Greenpeace a usé dans son recours d'arguments de procédure et de fond. Sur le fond, l'association a estimé que le principe de précaution n'était pas respecté en soulignant les risques liés à la dissémination du gène de résistance à l'ampicilline vers des bactéries pathogènes des animaux et

${ }^{64}$ Greenpeace (2003), «Greenpeace installe un piquet de manifestants à côté du champ d'essai», communiqué de presse du 28.2.2003, url: http://info.greenpeace.ch/fr/genie/communiques/ cp_280203_epfz

${ }_{65}$ Greenpeace (2003), «Action de Greenpeace à Lindau - la résistance grandit plus rapidement », communiqué de presse du 6.3.2003, url: http://info.greenpeace.ch/fr/genie/communiques/ cp_060303_epfz

${ }_{66}$ Voir: Greenpeace Suisse (2002), « Greenpeace annonce la résistance à la décision du Conseil fédéral concernant le blé transgénique», 13.09.2002, url: info.greenpeace.ch/fr/genie/Pressreleases/ cp_130902_ble 
des hommes. La seconde partie de l'argumentaire est plus intéressante de notre point de vue, car l'association de protection de l'environnement en profite pour élargir les termes du débat. En autorisant la culture de ce maïs transgénique, le Ministère de l'agriculture a, selon elle, privilégié des intérêts économiques particuliers. D'après l'association, cette mesure «favorise en effet l'agriculture intensive au détriment de l'agriculture familiale et biologique, et les multinationales de l'agrochimie et des semences au détriment des PME françaises » (Joly et al., 2001, p. 40). Est évoqué en effet le risque de contamination, par pollinisation, des champs voisins de maïs non transgénique, problème d'autant plus grave dans le cadre de l'agriculture biologique, les agriculteurs pouvant être tenus de garantir l'absence de contamination génétique. Greenpeace considère également le risque d'apparition de pyrales résistantes à la toxine $\mathrm{Bt}$. Si ce risque est gérable pour les agriculteurs qui utilisent des produits pesticides, il peut par contre avoir de graves conséquences pour les agriculteurs biologiques. Un tel événement priverait en effet ces derniers «du seul insecticide autorisé par les cahiers des charges de l'agriculture biologique» (Joly et al., 2000, p. 40).

Quant à Ecoropa, si elle s'est associée à Greenpeace et la CP dans la campagne visant à interdire le maïs transgénique de Novartis en France en 1998, elle a peu mené d'actions directes tournées vers le grand public. Sa force réside davantage dans sa capacité à mobiliser les scientifiques et à construire des réseaux dans ce milieu. Elle est par exemple à l'origine du lancement au mois de mai 1996 d'un appel aux scientifiques, sous forme de pétition, demandant un moratoire interdisant la commercialisation d'OGM pour l'alimentation. Une large part de l'argumentation d'Ecoropa se situe toutefois sur le terrain scientifique ou réglementaire (Joly et al., 2000, p. 57). Sur le plan scientifique, elle milite pour endiguer le développement rapide des OGM afin de donner aux chercheurs et aux instances réglementaires plus de temps pour mieux évaluer les impacts éventuels pour l'environnement et la santé, notamment la question de la transmission du gène de résistance aux antibiotiques et ses implications en termes de santé ou encore l'absence d'évaluation de la nature et de la toxicité des métabolites secondaires des herbicides comme le Round-up. Sur le plan réglementaire, Ecoropa relève que la réglementation en vigueur dans les pays de l'OCDE a été construite par les promoteurs du génie génétique. Dans ce sens, il ne peut y avoir de contrôle sur les OGM que dans la mesure où il n'introduit pas de contraintes supplémentaires sur le commerce des OGM (Joly et al., 2000, p. 57).

Quant à la Confédération paysanne, elle a choisi la stratégie de la «démonstration» et s'y est tenue. En n'hésitant pas à situer son action dans l'illégalité ${ }^{67}$, elle a dénié toute légitimité aux autorités officiellement habilitées à juger les demandes de disséminations expérimentales ou commerciales. Selon nous, c'est

Sur le plan de la légitimité, si des actions impliquant la destruction du bien d'autrui sont à même de choquer, cette question se pose différemment lorsqu'il s'agit de défendre un «bien supérieur», qu'il y a urgence et qu'il s'agit de la seule façon de se faire entendre. A ce titre, la décision du gouvernement de Lionel Jospin d'autoriser le maïs Bt de Novartis au mois de novembre 1997 revient à court-circuiter une autre décision prise simultanément: celle de lancer un débat public autour des OGM au mois de juin 1998 par la tenue d'une Conférence de citoyens. Pour les antiOGM, ce débat ressemble fâcheusement à une mesure cosmétique ayant davantage pour but d'avaliser une décision que de la discuter. 
la radicalité de cette stratégie qui lui a permis, ne serait-ce que temporairement, d'imposer son cadre de référence à d'autres arènes, que ce soient l'arène politique, scientifique ou l'arène de régulation. Elle a ainsi réussi à faire éclater le cadrage étroit de l'expertise - jusque-là cloisonné aux risques sanitaires et environnementaux - et à inclure dans le débat public la qualité des aliments, l'autonomie des paysans du Nord et du Sud, les aspects éthiques mais aussi socio-économiques du brevetage du vivant, ainsi que le risque, jusqu'alors passé sous silence, de voir les disséminations provenant de champs expérimentaux contaminer les cultures traditionnelles rendant une cohabitation entre cultures OGM et non OGM à terme illusoire (Heller, 2003, p. 8). Si les militants paysans ont réussi dès 1999 à imposer progressivement auprès de l'opinion publique l'équation $« \mathrm{OGM}=\mathrm{MacDo}=$ mondialisation », et donc à leur donner une connotation péjorative, c'est en partie grâce à l'épisode de Seattle. Bové y rencontre des paysans mexicains et indiens appartenant à la Via Campesina, une coalition d'organisations paysannes de petits et moyens agriculteurs à laquelle appartient la Confédération, ainsi que des syndicalistes paysans américains. Au centre de ce «mouvement paysan international», on constate une prise de conscience de l'existence d'une communauté d'intérêts entre le Nord et le Sud. Les OGM deviennent alors un symbole de rassemblement pour ceux qui luttent contre une agriculture productiviste, non respectueuse des équilibres sociaux.

Contrairement à la Confédération, Ecoropa et, dans une moindre mesure Greenpeace, ont toujours, consciemment ou non, abordé la question des OGM en termes de risques sanitaires ou environnementaux. Pour Greenpeace, c'est le cas lors des actions menées contre l'arrivée du colza transgénique en 1996 et contre le maïs Bt de Novartis. Si Greenpeace élargit son argumentaire lors de son recours au Conseil d'Etat, il n'en reste pas moins que c'est dans le cadre d'une procédure juridique et que l'impact médiatique de tels arguments est par définition limité. Dans ce contexte, la CP apparaît donc comme la seule organisation qui parvient à élargir le cadrage dominant en se basant non pas sur l'autorité judiciaire ou scientifique, qu'elle conteste, mais sur les médias et, à travers eux, le public français qu'elle prend à témoin lors de ses multiples «démonstrations». Si elle réussit ce tour de force, c'est, selon nous, parce que ses membres sont généralement des personnes qui n'ont pas été formées à la méthode scientifique. Travailleurs des champs et non des laboratoires, ils mobilisent une expertise basée sur leurs connaissances de la terre, de la culture alimentaire française, du mode de fonctionnement de la petite paysannerie. En devenant en quelque sorte eux-mêmes «experts» (Heller, 2002, p. 18-19), ils contestent aux scientifiques le privilège d'être les seuls à pouvoir tracer la frontière entre l'objectif et le subjectif, le vrai et le faux, le rationnel et l'irrationnel, en d'autres termes, entre ce qui relève de la science et ce qui relève de la société.

\section{CONCLUSIONS ET PERSPECTIVES}

En Suisse, durant toute la consultation ayant amené à la première décision de l'OFEFP en novembre 2001, le débat a porté presque exclusivement sur des arguments de type scientifique et juridique. L'OFEFP a justifié sa décision en se basant sur des considérations scientifiques et elle est en cela restée fidèle au cadrage de 
l'expertise fourni par les instances consultées. Le recours de l'EPFZ auprès du DETEC a élargi le débat à des arguments juridiques, mais ce n'est que lorsque diverses associations se sont mobilisées pour protester contre l'acceptation du recours de l'EPFZ et l'autorisation de la dissémination que d'autres cadres de référence ont fait irruption dans le débat. Mais le recours des opposants à l'essai a de nouveau inscrit le règlement de cette affaire dans le cadre d'une procédure judiciaire et le débat s'est restreint aux seuls arguments scientifiques et juridiques. Bien que tous les types d'arguments aient été présents au cours de cette controverse, l'écrasante majorité des points débattus, étudiés et confrontés portaient toutefois sur des questions relevant des arènes juridiques et scientifiques. Qui plus est, bien qu'évoqués, les arguments socio-économiques, éthiques et politiques n'ont jamais été explicitement pris en compte pour fonder une décision. En d'autres termes, ils n'ont pas réussi à s'imposer dans une arène concurrente, car il n'existe pas à l'heure actuelle en Suisse un cadre législatif et administratif qui permette de prendre en compte des arguments autres que scientifiques et juridiques.

En France, la société civile a réussi, grâce à la stratégie de la «démonstration », à introduire dans le débat sur les disséminations des arguments socio-économiques, éthiques et politiques. La manière dont les opposants se sont mobilisés semble avoir joué un rôle central dans la tournure donnée au débat. Il est en effet permis de penser que le facteur déterminant ayant permis un élargissement du débat et un décloisonnement de l'expertise a bel et bien été la stratégie de la Confédération paysanne en collaboration avec d'autres associations. Il ne faut toutefois pas oublier que le choix de cette stratégie n'est pas totalement indépendant mais découle de la structure politique française. La démocratie représentative et la centralisation ne permettent pas la prise en compte des intérêts des groupes concernés comme le font les droits populaires en Suisse. Du coup, les risques se «négocient» de façon moins institutionnelle qu'en Suisse (Audétat et al., 2004). Mais il ne faut pas surestimer les résultats de cette stratégie. Aujourd'hui les essais expérimentaux en France ont repris (on recense 17 autorisations pour 2003) ${ }^{68}$, et le moratoire européen a récemment été levé ${ }^{69}$, alors qu'en Suisse, seul l'essai du blé kp4 a finalement été autorisé, le premier depuis douze ans. Par ailleurs, l'expertise semble également avoir repris ses droits en France moyennant quelques réformes. On peut citer la création du comité de Biovigilance qui a pour tâche de surveiller les effets des plantes transgéniques sur l'environnement après l'autorisation de la mise sur le marché. L'originalité de ce comité tient en partie à sa composition, des associations opposées aux OGM comme Greenpeace et Ecoropa ayant l'opportunité de faire valoir leurs arguments lors des discussions sur les protocoles de recherche (notamment sur les risques potentiels que l'on peut envisager, sur la manière de les étudier) (Joly et al., 2000, pp. 44-45). La composition de la Commission du Génie Biomoléculaire (CGB) a également changé, faisant une place plus grande à des scientifiques connus pour leurs positions anti-OGM

68 Site d'information sur les OGM du Ministère de l'agriculture: http://www.ogm.gouv.fr/experimentations/decisions/decisions_suite2_2003.htm

69 L'autorisation de commercialisation dans l'Union Européenne du mais doux Bt-11 de Syngenta le 19 mai 2004 signe la fin du moratoire initié en juin 1999. 
(Joly et al., 2000, pp. 44-45). De manière similaire en Suisse, la décision de l'OFEFP d'autoriser finalement la dissémination du blé KP4 peut être lue comme le rétablissement de la continuité entre expertise et décision (ou entre évaluation et gestion des risques pour adopter une autre terminologie). La décision du DETEC a ainsi fait office d'avertissement pour l'autorité de régulation, l'expertise retrouvant une place centrale qu'elle n'a du reste jamais véritablement perdue.

Toutefois, en ce qui concerne la Suisse, un changement important pourrait venir du côté de la nouvelle loi fédérale sur l'application du génie génétique au domaine non humain (LGG) qui est entrée en vigueur le $1^{\text {er }}$ janvier 2004. Cette loi fixe en effet des objectifs beaucoup plus larges que ceux visés par la législation actuelle. En plus de la protection de l'être humain, des animaux et de l'environnement contre les abus en matière de génie génétique, la LGG a également pour but de veiller à ce que les applications du génie génétique servent l'être humain, les animaux et l'environnement ${ }^{70}$. De manière plus précise, la LGG vise bien sûr à protéger la santé et la sécurité de l'être humain, des animaux et de l'environnement et à conserver à long terme la diversité biologique et la fertilité du sol. Mais elle introduit également des objectifs nouveaux: garantir l'intégrité des organismes vivants, permettre le libre choix des consommateurs, empêcher la fraude sur les produits, encourager l'information du public et, enfin, tenir compte de l'importance de la recherche scientifique dans le domaine du génie génétique pour l'être humain, les animaux et l'environnement ${ }^{71}$. Les conditions et la procédure d'autorisation relative aux disséminations expérimentales ne sont pas directement précisées dans la LGG. Toutefois, les ordonnances sur les disséminations dans l'environnement et sur l'utilisation confinée étant déjà relativement précises, il ne devrait donc vraisemblablement pas y avoir de nouvelle ordonnance spécifique à la LGG mais une adaptation de celles déjà existantes ${ }^{72}$. La LGG constitue donc une réelle amélioration par rapport à la Loi sur la protection de l'environnement (LPE). Elle permet de prendre en compte des dimensions allant au-delà des dimensions strictement scientifiques relatives aux risques environnementaux et sanitaires. En ce sens, on peut espérer qu'elle contribue à élargir le débat public et qu'elle permette de rétablir une clarification des rôles entre les experts chargés de l'évaluation des risques (la CSFB) et l'instance de décision en charge de leur gestion (l'OFEFP).

Enfin, l'initiative «Pour des aliments produits sans manipulations génétiques» (initiative dite «Stop OGM»), qui a été déposée en septembre 2003 après avoir récolté plus de $120^{\prime} 000$ signatures, devra être soumise à la votation populaire. Bien qu'elle ne s'applique pas à la recherche, et qu'elle ne concerne donc pas directement les demandes de disséminations expérimentales, cette initiative pose des questions qui méritent selon nous d'être débattues et qui vont probablement permettre d'élargir le débat public. En proposant la mise en place d'un moratoire de 5 ans sur l'utilisation d'OGM dans l'agriculture, cette initiative sera vraisemblablement l'occasion d'approfondir des problématiques aussi essen-

Art. 1.1.lit. a et b LGG

Art.1.2.lit a à g LGG

OFEFP, «Sécurité biologique, prévenir vaut mieux que guérir», Fiche d'information journée internationale de l'environnement, 5 juin 2003. 
tielles que la place des OGM dans l'agriculture Suisse, la possibilité de coexistence entre la filière traditionnelle et la filière OGM, ou encore la question des OGM dans l'alimentation.

\section{LISTE DES ACRONYMES}

AWEL Service spécialisé pour la sécurité biologique du Service des déchets, de l'eau, de l'énergie et de l'air

CETIOM Centre technique interprofessionnel des oléagineux métropolitains

CENH Commission fédérale d'éthique dans le domaine non humain

CFSB Commission fédérale d'experts pour la sécurité biologique

CGB Commission de génie biomoléculaire

CIRAD Centre de coopération internationale en recherche agronomique pour le développement

CNRS Centre national de la recherche scientifique

CP Confédération paysanne

DETEC Département fédéral de l'environnement, des transports, de l'énergie et de la communication

EPFZ Ecole polytechnique fédérale de Zurich

FNS Fonds national pour la recherche scientifique

FNSEA Fédération nationale des syndicats des exploitants agricoles

INRA Institut national de recherche agronomique

IRD Institut de recherche pour le développement

LGG Loi sur le génie génétique

LPE Loi sur la protection de l'environnement

OCDE Organisation de coopération et de développement économiques

ODE Ordonnance sur les disséminations dans l'environnement

OFAG Office fédéral de l'agriculture

OFEFP Office fédéral de l'environnement, des forêts et du paysage

OFSP Office fédéral de la santé publique

OGM Organisme génétiquement modifié

OMC Organisation mondiale du commerce

OUC Ordonnance sur l'utilisation confinée

OVF Office vétérinaire fédéral

PPB Programme prioritaire biotechnologie 


\section{Chronologie des événements}

\begin{tabular}{|c|c|}
\hline 15 novembre 2000 & $\begin{array}{l}\text { Demande d'autorisation pour une dissémination expérimentale } \\
\text { de blé génétiquement modifié pour résister à la carie (maladie } \\
\text { fongique) par l'Institut de biologie végétale de l'EPFZ. La } \\
\text { période envisagée pour l'essai est février } 2002 \text {-avril } 2003 \text {. Les } \\
\text { objectifs annoncés de cette dissémination sont l'évaluation de } \\
\text { la résistance à la carie du blé et l'analyse des interactions avec } \\
\text { les organismes non cible. La demande étant incomplète, l'Of- } \\
\text { fice fédéral de l'environnement, des forêts et du paysage } \\
\text { (OFEFP) retourne le dossier. }\end{array}$ \\
\hline 4 janvier 2001 & $\begin{array}{l}\text { La demande d'autorisation complétée est déposée auprès de } \\
\text { l'OFEFP. }\end{array}$ \\
\hline 13 février 2001 & $\begin{array}{l}\text { Publication de la demande d'autorisation dans la Feuille fédé- } \\
\text { rale. }\end{array}$ \\
\hline Février 2001 & $\begin{array}{l}\text { Diverses informations complémentaires sont demandées à } \\
\text { l'EPFZ. }\end{array}$ \\
\hline 21 février 2001 & $\begin{array}{l}\text { Soirée d'information au public dans la commune de Lindau } \\
\text { organisée par l'OFEFP et par l'équipe du Prof. Sautter de l'Ins- } \\
\text { titut de biologie végétale de l'EPFZ. }\end{array}$ \\
\hline Juillet/octobre 2001 & l'EPFZ fourni les compléments d'information demandés. \\
\hline 20 novembre 2001 & La demande est refusée par l'OFEFP. \\
\hline 27 novembre 2001 & $\begin{array}{l}\text { Cinq membres de la CFCB démissionnent suite à la décision de } \\
\text { l'OFEFP. }\end{array}$ \\
\hline 31 décembre 2001 & $\begin{array}{l}\text { L'EPFZ fait recours auprès du Département Fédéral de l'Envi- } \\
\text { ronnement, des Transports, de l'Energie et de la Communica- } \\
\text { tion (DETEC) contre la décision de l'OFEFP. }\end{array}$ \\
\hline 12 septembre 2002 & $\begin{array}{l}\text { Le DETEC admet le recours de l'EPFZ. L'autorisation de dissé- } \\
\text { mination expérimentale est accordée mais le dossier est renvoyé } \\
\text { à l'OFEFP pour qu'il en fixe précisément les conditions. }\end{array}$ \\
\hline 20 décembre 2002 & $\begin{array}{l}\text { L'OFEFP autorise la dissémination de blé génétiquement } \\
\text { modifié de l'EPFZ sous certaines conditions. }\end{array}$ \\
\hline 27 janvier 2003 & $\begin{array}{l}\text { Le Conseil fédéral confirme la réglementation actuelle selon } \\
\text { laquelle l'OFEFP est l'organe compétent pour délivrer les auto- } \\
\text { risations de disséminations d'OGM. }\end{array}$ \\
\hline 29 janvier 2003 & $\begin{array}{l}\text { Un recours est déposé auprès du DETEC contre la décision de } \\
\text { l'OFEFP du } 20 \text { décembre } 2002 \text { par IP-Suisse, des riverains du } \\
\text { site d'essai de Lindau organisés en groupe de travail «Lindau } \\
\text { contre le blé transgénique» et Greenpeace. }\end{array}$ \\
\hline 20 février 2003 & $\begin{array}{l}\text { Le DETEC retire l'effet suspensif du recours déposé par IP- } \\
\text { Suisse, les riverains et Greenpeace le } 29 \text { janvier } 2003 \text {. }\end{array}$ \\
\hline 28 février 2003 & $\begin{array}{l}\text { L'OFEFP met en place un groupe d'accompagnement chargé } \\
\text { de surveiller le bon déroulement de l'essai de blé transgénique } \\
\text { de l'EPFZ. }\end{array}$ \\
\hline
\end{tabular}




\begin{tabular}{|l|l|}
\hline 3 mars 2003 & $\begin{array}{l}\text { IP-Suisse, les riverains de Lindau et Greenpeace, et font recours } \\
\text { contre la décision du DETEC du } 21 \text { février 2003 }\end{array}$ \\
\hline 12 mars 2003 & $\begin{array}{l}\text { Le Tribunal fédéral accepte le recours et l'effet suspensif du } \\
\text { recours du 29 janvier 2003 est rétabli. }\end{array}$ \\
\hline 28 mars 2003 2001 & $\begin{array}{l}\text { Entrée en vigueur de la loi fédérale sur l'application du génie } \\
\text { génétique au domaine non humain (Loi sur le génie génétique, } \\
\text { LGG) }\end{array}$ \\
\hline 3 avril 2003 & $\begin{array}{l}\text { Une pétition comptant environ 700 signatures récoltées dans la } \\
\text { commune de Lindau et ses environs est envoyée à l'OFEFP. }\end{array}$ \\
\hline 13 juin 2003 & $\begin{array}{l}\text { L'EPFZ demande à ce que toute la procédure d'autorisation soit } \\
\text { reprise à zéro. Les opposants à l'essai appuient cette demande. }\end{array}$ \\
\hline 22 juillet 2003 & $\begin{array}{l}\text { Le DETEC approuve le recours des associations de riverains et } \\
\text { de défense de l'environnement. La procédure d'évaluation } \\
\text { devra être reprise depuis le début. }\end{array}$ \\
\hline $\begin{array}{l}\text { l'EPFZ publie une nouvelle demande d'autorisation pour la } \\
\text { dissémination expérimentale de blé transgénique résistant à la } \\
\text { carie. La période d'essai envisagée est mars 2004-avril 2005. }\end{array}$ \\
\hline 30 octobre 2003 & $\begin{array}{l}\text { L'OFEFP autorise l'essai en plein champ de l'EPFZ concernant } \\
\text { le blé KP4. }\end{array}$ \\
\hline 27 novembre 2003 février 2004 & $\begin{array}{l}\text { Greenpeace et IP Suisse recourent auprès du DETEC contre } \\
\text { l'autorisation délivrée par l'OFEFP le 30 octobre 2003. }\end{array}$ \\
\hline 18 mars 2004 & \begin{tabular}{l} 
Le DETEC rejette le recours de Greenpeace et IP Suisse. \\
Le blé transgénique kp4 est semé par l'EPFZ sur la commune \\
\hline
\end{tabular} \\
\hline
\end{tabular}

\section{BIBLIOGRAPHIE}

Audétat M., Kaufmann A., Joseph C. et Leresche J.-P. (2004), «Usages et limites de l'expertise dans la négociation des risques climatiques et biotechnologiques en Suisse», in P. Warin et al. (Eds.), Le recours aux experts, CERAT, Grenoble (à venir).

Barry A. (2001), Political Machine. Governing a technological society, London \& New York, The Athlone Press.

Bonneuil C. (2001), «La question des cadrages de l'expertise en situation d'incertitude», in Actes du colloque de 1'Institut Pasteur, «Maîtrise des risques: prévention et principe de précaution », 6 novembre.

Callon M., Lascoumes P. et Barthe Y. (2001), Agir dans un monde incertain: essai sur la démocratie technique, Paris, Editions du Seuil.

Callon M. (2003), Note de lecture sur l'ouvrage de Barry A. «Political machine. Governing a technological society», Cosmopolitiques, $\mathrm{n}^{\circ} 3$ février.

Callon M., Lascoumes P. et Barthe Y. (2003), «La démocratie dialogique casse-t-elle des briques?», Cosmopolitiques, $\mathrm{n}^{\circ} 3$ février.

Confédération paysanne, Communiqué de novembre 2003, url: http ://www.confederationpaysanne.fr/ anapro/liste.htm\#ogm

De Cheveigné S., Boy D. et Galloux J.-C. (2002), Les biotechnologies en débat, Paris, éditions Balland. 
De Sadeleer N. et Noiville C. (2001), «La gestion des risques écologiques et sanitaires à l'épreuve des chiffres: Le droit entre enjeux scientifiques et politiques », Revue de droit de l' Union Européenne, 02 .

Heller C. (2002), «From scientific risk to paysan savoir-faire: peasant expertise in the French and global debate over GM crops », Science as Culture, vol.11, number 1.

Joly P.-B. et Assouline G. (June 2001), Assessing debate and participative technology assessment in Europe, Grenoble, INRA and QAP Decision.

Joly P.-B., Assouline G., Kreziak D., Lemarie J., Marris C., Roy A. (janvier 2000), L'innovation controversée : le débat public sur les OGM en France, Grenoble, INRA (Collectif sur les Risques, la Décision et l'Expertise).

Joly P.-B. et Marris C. (2002), La trajectoire d'un problème public: une approche comparée du cas des OGM en France et aux Etats-Unis, in Gilbert C., «Risques collectifs et situations de crise: apports de la recherche en sciences humaines et sociales », Paris, L'Harmattan.

Kempf H. (2003), La guerre secrète des OGM, Paris, Seuil (L'histoire immédiate).

Pingaud D. (2002), La longue marche de José Bové, Paris, Seuil.

Roy A. (2001), Les experts face au risque: le cas des plantes transgéniques, Paris, PUF. 